\title{
Optimal allocation of HVDC interconnections for exchange of energy and reserve capacity services
}

Delikaraoglou, Stefanos; Pinson, Pierre

\section{Published in:}

Energy Systems

Link to article, DOI:

$10.1007 / \mathrm{s} 12667-018-0288-6$

Publication date:

2019

Document Version

Early version, also known as pre-print

Link back to DTU Orbit

Citation (APA):

Delikaraoglou, S., \& Pinson, P. (2019). Optimal allocation of HVDC interconnections for exchange of energy and reserve capacity services. Energy Systems, 10(3), 635-675. https://doi.org/10.1007/s12667-018-0288-6

\section{General rights}

Copyright and moral rights for the publications made accessible in the public portal are retained by the authors and/or other copyright owners and it is a condition of accessing publications that users recognise and abide by the legal requirements associated with these rights.

- Users may download and print one copy of any publication from the public portal for the purpose of private study or research.

- You may not further distribute the material or use it for any profit-making activity or commercial gain

- You may freely distribute the URL identifying the publication in the public portal 


\title{
Optimal allocation of HVDC interconnections for exchange of energy and reserve capacity services
}

\author{
Stefanos Delikaraoglou • Pierre Pinson
}

Received: date / Accepted: date

\begin{abstract}
The increasing shares of stochastic renewables bring higher uncertainty in power system operation and underline the need for optimal utilization of flexibility. However, the European market structure that separates energy and reserve capacity trading is prone to inefficient utilization of flexible assets, such as the HVDC interconnections, since their capacity has to be ex-ante allocated between these services. Stochastic programming models that co-optimize day-ahead energy schedules with reserve procurement and dispatch, provide endogenously the optimal transmission allocation in terms of minimum expected system cost. However, this perfect temporal coordination of trading floors cannot be attained in practice under the existing market design. To this end, we propose a decision-support tool that enables an implicit temporal coupling of the different trading floors using as control parameters the interregional transmission capacity allocation between energy and reserves and the area reserves requirements. The proposed method is formulated as a stochastic bilevel program and cast as mixed-integer linear programming problem, which can be efficiently solved using a Benders decomposition approach that improves computational tractability. This model bears the anticipativity features of a transmission allocation model based on a pure stochastic programming formulation, while being compatible with the current market structure. Our analysis shows that the proposed mechanism reduces the expected system cost and thus can facilitate the large-scale integration of intermittent renewables.
\end{abstract}

S. Delikaraoglou

EEH-Power Systems Laboratory

Swiss Federal Institute of Technology (ETH)

Zurich, Switzerland

E-mail: stde@ee.eeh.ethz.ch

P. Pinson

Department of Electrical Engineering

Technical University of Denmark

Kgs. Lyngby, Denmark

E-mail: ppin@dtu.dk 
Keywords Electricity markets - High Voltage Direct Current (HVDC) · Transmission capacity allocation · Reserve capacity · Stochastic programming · Bilevel programming

\section{Introduction}

Over the last decades, the appeal for a more sustainable power system has motivated the large-scale integration of renewable energy sources (RES). This ongoing transformation is currently taking place in a market environment that was initially conceived to serve fossil-fueled generation. However, the ability of the current market mechanisms to mobilize the necessary flexibility to contain the variable and partially predictable RES production is currently called into question [43], [2].

Despite the fact that regional electricity markets exhibit some differences in terms of regulation and trading arrangements, they still built upon the same fundamental principles and organizational structure [48], [45]. The main components of existing electricity markets are the day-ahead (forward) and the balancing trading floors, which are typically settled independently through sequential auctions. The day-ahead market is a purely financial process, with obligation for physical delivery, that usually takes place 12-36 hours in advance of actual operation to establish generation schedules and derive electricity prices. Getting close to real-time operation, any deviations from the day-ahead schedule need to be compensated through the balancing market in order to maintain power balance between demand and supply. In this settlement process uncertain RES production is dispatched to a single-valued forecast, typically the conditional expectation of the predictive distribution. Based on this deterministic view of the stochastic RES production, current market design suffers from imperfect temporal coordination of day-ahead and balancing trading floors, since the day-ahead schedule is myopic about the full range of real-time system uncertainties.

Day-ahead and balancing markets pertain to the amount of energy actually delivered to the network and thus constitute a form of energy-only auctions. Apart from energy, another important tradable component involved in power system operation is operating reserves. This component refers to the capacity withdrawn from the day-ahead market to be available for provision of balancing services during real-time operation. In the European context, operating reserves are procured through independent capacity markets, while the US approach employs a multi-commodity auction that co-optimizes these two services. In both cases, reserve requirements must be provided as input to the reserve capacity market and thus must be exogenously determined by the system operator. Existing reserve determination methodologies are mainly based on ad-hoc heuristic approaches [15], [36], [21], as for instance the N-1 criterion which guarantees that the system will be able to withstand the outage of the largest unit or transmission line. However, as the shares of stochastic RES 
increase, these deterministic criteria might become obsolete since the main driver of reserve requirements is renewables' forecast errors [34].

In order to improve day-ahead hedging opportunities against real-time prediction errors, recent literature proposes alternative dispatch approaches that embrace a probabilistic view of system uncertainties. Based on a stochastic programming framework [6], day-ahead and balancing stages are co-optimized to minimize expected system cost [25], [40], [47], [52] accounting for the intertemporal arbitrage between those stages. These models attain perfect temporal coordination, performing an endogenous trade-off between the value of flexibility in the day-ahead and balancing stages to determine the generation and transmission set-points, instead of relying on deterministic rules similar to reserve requirements. However, these frameworks cannot be directly applied to real-life market-based operations, since they are not compatible with the existing market structures. As a result, the decision maker, i.e., system operator, has no guarantee that by applying the solution of a stochastic dispatch model to the current market setup will actually reproduce the optimal results. Nonetheless, in the context of electricity markets these models can provide useful insights to decision makers and establish an optimal benchmark to quantify the efficiency gap of the prevailing market architecture.

Renewable generation exhibits particular spatial correlation patterns. Especially in the European system, renewable production has substantial impact on market prices and on inter-regional power flows [60], since the correlation scale is of the same order as the size of regional market areas [28]. Despite the recent establishment of the European internal electricity day-ahead market, the provision and activation of reserves remain mainly an exclusive responsibility of regional system operators. In this regard, European electricity market lacks complete spatial coordination with respect to reserve capacity and balancing trading floors. A thorough analysis of the issues related to cross-border exchange of reserves and transmission capacity allocation is provided in [26].

These considerations open the discussion towards integrated reserve capacity and balancing markets that enable access to system-wide flexible resources. To enhance spatial coordination in reservation and deployment of reserves, flexible transmission-side infrastructure is a major prerequisite. In this vein, High Voltage Direct Current (HVDC) interconnections can provide this necessary flexibility given their increased controllability compared to prevailing AC grids [3]. It is envisioned that HVDC lines will play a major role as crossborder interconnections in the European power system and given their ability to shift rapidly the power flow direction, HVDC links can broaden the set of available recourse actions during real-time operation. Nonetheless, in view of separate reserve and energy trading, the efficient allocation of interconnection capacities between these services is a major prerequisite for the establishment of well-functioning integrated reserve capacity and balancing markets.

Acknowledging the restrictions of the current market framework, the objective of this paper is to propose decision-support mechanisms that improve spatial market coordination. These mechanisms aim at promoting the integration of reserve capacity and balancing markets, addressing the issue of 
inter-area transmission capacity allocation between energy and reserves. In the context of this work, we use the term control parameters to characterize market quantities that are decision variables of the market operator prior to market clearing, but obtain fixed values during the settlement process. The rationale of our approach is to select properly those parameters in order to steer implicitly the market outcome closer to the stochastic ideal dispatch. In our framework, these control parameters are the inter-area transmission allocation and the regional reserve requirements, that both enter market clearing as fixed values but affect directly all trading floors, since they control the amount of generation and transmission capacity set aside from energy provision to be available for balancing.

First, we use as control parameter the share of tie-line capacity released at the reserve procurement phase and we establish a systematic method to find the optimal value of this parameter. The proposed model is formulated as a stochastic bilevel programing problem that explicitly accounts for the temporal coordination of reserve capacity, day-ahead and balancing markets as well as for the spatial characteristics of uncertainty through a properly constructed scenario set. To further improve market efficiency in terms of dimensioning and procurement cost, we formulate a more advanced version of this model using as additional degree of freedom the regional reserve requirements. Both models are compatible with the existing sequential market architecture and allow us to consider mixed AC and DC grids spanning multiple areas. Mathematically, these stochastic bilevel programming models are reformulated as mixed-integer linear programs that can be readily solved using branch-and-bound algorithms. To improve computational tractability, we exploit the particular structure of these problems and we formulate an iterative solution scheme based on Benders decomposition that guarantees convergence to the optimal solution.

Our bilevel model shares some similarities with [41], since both works use stochastic bilevel programing to impose mathematically the merit-order of various trading floors according to the sequential market design. However, our work uses this structure to design a decision-support tool that is fully compatible with the current market structure. On the contrary, [41] suggests a new market-clearing process that alters day-ahead merit-order and dispatches stochastic production to a value that is generally different from its conditional expectation. In addition, our work considers the full sequence of trading floors of the European market in multi-area setup, as opposed to [41] that focuses on an energy-only single-area setup disregarding reserve capacity markets.

The rest of this paper is organized as follows. Section 2 discusses the issues related to inter-area exchange of reserve capacity services in Europe and gives an overview of the different coordination arrangements and transmission allocation methods. Section 3 describes the mathematical structure of the transmission allocation models used in this work and presents our solution approach that is based on a Benders decomposition scheme. Section 4 presents and discusses the results of an illustrative example and a more realistic case study. Finally, Section 5 concludes the paper and gives suggestions for future work. 


\section{Context and current issues regarding cross-border coordination} of reserve capacity services

This section provides an overview of the regulatory aspects related to the exchange of balancing services among multiple regions in the European system. These issues refer to coordination arrangements during the reserve procurement and deployment phases as well as to different approaches for allocation of inter-regional transmission capacity for the exchange of balancing services.

\subsection{State of affairs}

Historically, cross-border transmission capacity was used to ensure synchronization and security of supply in case of contingencies, whereas the current regulatory framework aims to maximize the utilization of cross-border transmission for day-ahead energy trade. This requirement stems from the implicit assumption that the value of interconnections is higher for energy than for exchange of balancing services. However, day-ahead energy prices do not always reflect the scarcity and value of transmission for every operational time frame, e.g., balancing, especially in the presence of near-zero marginal cost renewable generation and limited real-time flexibility.

Considering the scarce flexible generation, reserve capacity and balancing markets are more prone to market power issues due the limited liquidity. Hence, pooling available balancing resources from multiple areas will increase competition and reduce the socio-economic cost of balancing [30]. In this vein, the establishment of cross-border balancing mechanisms can reduce RES curtailment [42] and improve system's reliability level [35].

The authors in [5] estimate the benefits of coordinated balancing operations of Central Western European power system (Belgium, France, Germany and the Netherlands) around $€ 25$ million per year. Likewise, the integration of the balancing and procurement markets of Northern Europe, Germany and the Netherlands are estimated to $€ 400$ million per year [22]. Despite these significant benefits, the number of existing cross-border cooperation arrangements for balancing purposes is very limited. An exceptional example is the Skagerrak 4 HVDC interconnector between Norway and Western Denmark, in which $15 \%$ of transmission capacity is permanently allocated for exchange of ancillary services [16]. This initiative aims at capturing the complementarity between the hydro-based Norwegian and the thermal/wind-based Danish power systems. However, as shown in previous work [13], this static allocation of transmission capacity is sub-optimal, since the true optimum varies dynamically depending on generation, load and system uncertainties.

Owing to the inefficiency of a priori interconnection reservation, the initial position of the European regulator was that cross-border transmission reservation shall be forbidden unless "...TSOs can demonstrate that such reservation would result in increased overall social welfare..." [1]. In practice, the main obstacle against an integrated European balancing market stems from the sep- 
arate trading of energy and reserve capacity commodities. Given this market structure, the establishment of cross-border balancing mechanisms requires the ex-ante allocation of interconnection capacity for each of these services. The Hasle-pilot [54] project implemented in 2014 by the Norwegian and Swedish TSOs has shown that it is both possible and socio-economically beneficial to reserve capacity for reserves exchange. Today, the updated European regulatory framework [20] identifies these opportunities and proposes three alternative processes for the allocation of cross-border transmission between energy and reserves. The following sections outline the main degrees of coordination in the procurement and activation of reserves, the different approaches for the allocation of interconnections as well as potential inefficiencies that may arise in practice.

\subsection{Degrees of coordination in procurement and activation of reserves}

Traditionally, regional system operators had to rely solely on their internal balancing resources to ensure the stable operation of their systems. However, moving towards an integrated balancing market, different arrangements with varying degrees of coordination may emerge regarding reserve procurement and activation processes [17],[19].

In terms of procurement, the two main coordination structures are reserve exchange and reserve sharing. The former setup refers to procurement of crossborder capacity to meet regional reserve requirements defined independently by each TSO. This coordination structure changes only the geographical distribution of reserves, i.e., more reserves are procured in cheap zones, since these can only be activated from the contracting TSO. Aiming to improve coordination in the procurement phase, reserve sharing allows multiple TSOs to account for the same reserves to meet their regional requirements. This setup requires coordinated dimensioning of reserves to prevent their depletion during real-time operation. Hence, reserve sharing enhances both dimensioning and procurement cost efficiency.

The two degrees of coordination in reserve activation are imbalance netting and exchange of balancing energy. Imbalance netting refers to exchange of counter imbalances between adjacent regions, before the activation of domestic reserves. This reduces the volumes of balancing energy and consequently the balancing costs. Exchange of balancing energy implies the establishment of a common merit-order list to ensure least-cost activation of reserves, irrespective of their location. This mechanism is currently implemented in the Nordic system, where all offers for manual up and down regulation are collected in a single pool that is referred to as the NOIS list (NOIS - Nordic Operational Information System) [8].

\subsection{Alternative approaches for cross-border transmission allocation}

The current European regulation identifies three main approaches for the allocation of cross-border transmission capacity between energy and reserves. In 
case of AC interconnections, the allocation should be determined by either cooptimized or market-based processes, while for DC interconnections capacity may be allocated based on economic efficiency analysis.

The most basic transmission capacity reservation method is based on economic efficiency analysis in which transmission allocation is performed according to an estimate of the reserve and energy bid spreads at the two ends of the interconnections. The capacity allocation of Skagerrak 4 HVDC interconnection, in which $100 \mathrm{MW}$ are reserved for exchange of ancillary services and $600 \mathrm{MW}$ are available for energy trade, was defined according to this method. Obviously, this approach is prone to price prediction errors that become an even more challenging issue as the shares of stochastic production increase [33].

At the other extreme, the most advanced allocation method follows a cooptimization process where TSOs and energy traders bid in the same market for the procurement of transmission capacity. This approach resembles an integrated market for energy, transmission and reserves that in theory yields the most efficient utilization of these services, being also free from price prediction errors. However, the practical implementation of this approach requires the establishment of a hybrid coupling model [29]. This is a prerequisite to maintain energy trading through implicit auctions, in compliance with the European 'Target Model', while allowing TSOs to bid for explicit transmission capacity procurement, based on reserve price-quantity offers at the two sides of the tieline. Despite its theoretical advantages, the complex arrangements involved in this method may hamper its actual application in the short-term future.

This work focuses on the third transmission allocation approach that follows a market-based reservation methodology. Under this scheme, TSOs reserve cross-border transmission capacity prior to day-ahead energy trading, based on submitted reserve offers and estimated difference of energy prices across interconnections. Although well aligned with current market structure, demonstrated also by its real-life implementation in the Hasle-pilot project, the market-based reservation method still suffers from some fundamental flaws that diminish its efficiency.

\subsection{Open issues and caveats of current transmission allocation models}

Despite that the processes described above constitute a systematic and transparent approach for tie-line capacity allocation, they disregard some essential aspects pertaining to market dynamics and to the uncertainty arising from short-term forecast errors of stochastic power generation. To facilitate understanding of potential caveats related to the existing market-based transmission allocation model, we consider a stylized power system that comprises areas 1 and 2 interconnected with a lossless transmission line. The following discussion focuses on the cross-border exchange of upward reserves, but the same reasoning applies also for downward reserves. We assume that the intra-area networks have infinite capacity and the two areas are connected through a 
tie-line with $100 \mathrm{MW}$ transmission limit. In each area $a$, the energy price $\lambda_{a}$ and the reserve capacity price $\varphi_{a}$ are described by the following inverse supply curves:

$$
\begin{aligned}
& \lambda_{a}\left(p_{a}\right)=\alpha_{a}\left(p_{a} \pm p^{e}\right)+\beta_{a}, \\
& \varphi_{a}\left(r_{a}^{+}\right)=\hat{\alpha}_{a}\left(r_{a}^{+} \pm r^{e}\right)+\hat{\beta}_{a},
\end{aligned}
$$

where $p_{a}$ and $r_{a}^{+}$denote the energy production and upward reserve capacity procured by each area, respectively. Energy exchange is denoted by $p^{e}$ and thus a positive sign in (1a) indicates energy import to this area, whereas a negative sign translates to energy export. A similar convention is used to model reserve exchange $r^{+}$. Each area faces an inelastic electricity demand $d_{a}$ and has to meet upward reserve capacity requirements equal to $R R_{a}^{+}$. The inverse supply curves (1a) and (1b) provide the required price in each area $a$, i.e., $\lambda_{a}$ and $\varphi_{a}$, to incentivize the provision of certain level of energy and reserve capacity, respectively. For this illustrative example, energy and reserve capacity inverse supply curves, shown in Figure 1, are described by linear functions and their coefficients $\left(\alpha_{a}, \beta_{a}\right)$ and $\left(\hat{\alpha}_{a}, \hat{\beta}_{a}\right)$ are provided in Table 1 , assuming that all generators offer their full capacity in the reserve market at $50 \%$ of their marginal cost.

Table 1: Coefficients of energy and reserve capacity demand functions, energy demand and upward reserve requirements in each area.

\begin{tabular}{ccccccc}
\hline Area & $\alpha_{a}$ & $\beta_{a}$ & $\hat{\alpha}_{a}$ & $\hat{\beta}_{a}$ & $d_{a}$ & $R R_{a}^{+}$ \\
\hline 1 & 0.2 & 20 & 0.1 & 0.5 & 200 & 40 \\
2 & 1 & 5 & 10 & 2.5 & 150 & 70 \\
\hline
\end{tabular}

According to the market-based reservation approach, the optimal capacity allocation of the interconnector is the point where the marginal value of transmission for day-ahead energy trade is equal to the marginal value for trade of reserves. From a short-term financial viewpoint, the marginal value MV of the transmission capacity for a specific service is equal to the price spread between the two areas, i.e., $\mathrm{MV}_{\lambda}=\lambda_{2}-\lambda_{1}$ and $\mathrm{MV}_{\varphi}=\varphi_{2}-\varphi_{1}$ are the marginal value of the tie-line for the exchange energy and reserves, respectively. For illustration purposes, these values are drawn in Figure 2 as $M_{\lambda}^{0}$ and $M_{\varphi}^{0}$, assuming that no exchange of neither energy nor reserves is allowed, i.e., each area has to cover its electricity load and reserve requirements using only intra-area resources. Based on the previous definitions, the optimal point $\mathrm{A}$ is found at the intersection of lines $\mathrm{MV}_{\lambda}$ and $\mathrm{MV}_{\varphi}$ as shown in Figure 2.

From this illustrative example, it becomes apparent that the accuracy of energy price spread predictions determines to a great extent the efficiency of this method. However, current implementations, e.g., [55], follow a persistent prediction approach assuming that the hourly energy price spread is equal to 

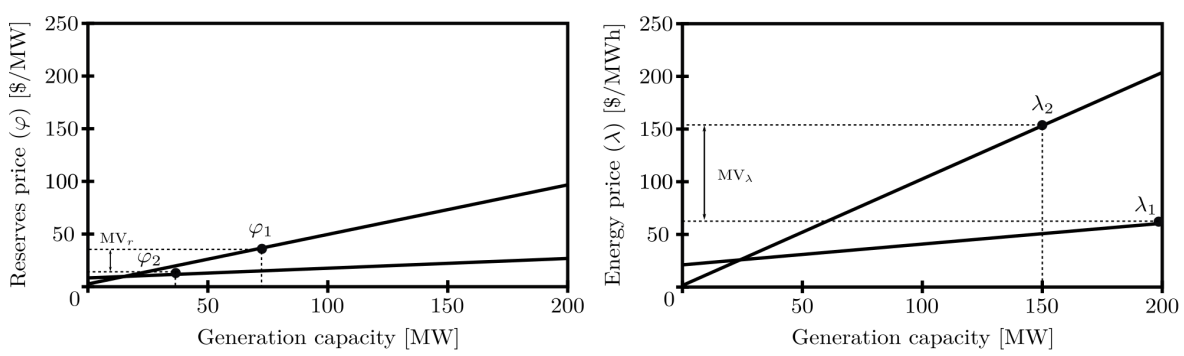

Fig. 1: Inverse supply curves for reserve capacity and energy.

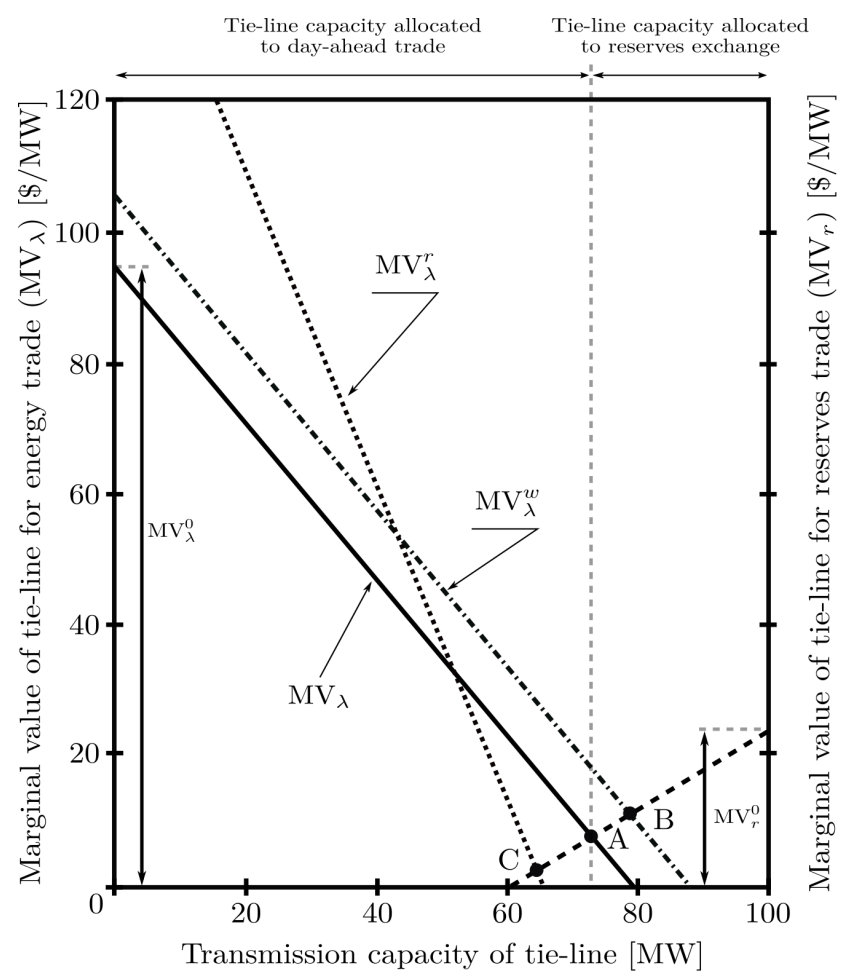

Fig. 2: Marginal value functions of tie-line capacity for energy and reserves trade.

the corresponding price difference of the previous week. Uncertainty in price predictions is reflected by ad-hoc criteria based on empirical knowledge of the TSOs. However, this simplified perception of market dynamics disregards three important factors that drive day-ahead energy prices.

The first factor pertains to the effect of stochastic production on the energy supply curve. Given the near-zero offers of renewable sources, e.g., wind power, day-ahead prices are directly affected by renewable predictions that tend to shift the supply curve inwards (outwards) in case of low (high) forecast produc- 
tion [32]. In the meantime, stochastic generation may differ significantly in the course of a week. However, this information is not captured by historical price spreads. To illustrate the detrimental impact of inadequate modeling of this merit-order effect, let us consider a variation of the previous illustrative example where stochastic production forecast in area 2 is decreased by $\Delta w=10$ MW. This renders a new energy supply function $\lambda_{2}^{w}\left(p_{2}\right)=\alpha_{2}\left(p_{2}-p^{e}-\Delta w\right)+\beta_{2}$ that changes the marginal value for energy trade to $M V_{\lambda}^{w}$ and consequently the optimal transmission allocation is now given by point B in Figure 2 .

Another important aspect that is not explicitly considered in this transmission allocation method is that energy and reserves act both as complements and as partial substitutes with respect to the available generation capacity [4]. Energy production is a prerequisite for the provision of downward reserves, whereas the procurement of upward reserves indicates that the generation unit operates at less than its full capacity. Furthermore these services are substitutional, given that the ability to deploy reserves, i.e., produce balancing energy, implies that this generation capacity is not available for energy provision at the day-ahead market. Returning to our initial example, the relationship between energy and upward reserves is encountered by adding the term $r_{a}^{+}$explicitly in the energy supply function as:

$$
\lambda_{a}^{r}\left(p_{a}\right)=\alpha_{a}\left(p_{a} \pm p^{e}+r_{a}^{+}\right)+\beta_{a}
$$

and taking into account that $r_{1}^{+}+r_{2}^{+}=R R_{1}^{+}+R R_{2}^{+}$. This modified supply function indicates that generation capacity assigned to provide upward reserves is actually withdrawn from the day-ahead energy market and thus more expensive generation is dispatched to meet electricity demand. In this case, the optimal allocation is shown in Figure 2 as the intersection of lines $\mathrm{MV}_{\lambda}^{r}$ and $\mathrm{MV}_{\varphi}$ at point $\mathrm{C}$ that differs significantly from the initial point A.

Being myopic about the structure of forecast uncertainty, the market-based allocation method is also flawed regarding the relationship of energy and reserve deployment. To illustrate the source of this inefficiency, we consider two predictive distributions for the normalized stochastic in-feed, i.e., values per unit (p.u.) of installed capacity, that are approximated by two scenario sets

$$
\begin{aligned}
& \mathcal{S}=\left\{0.9: \pi_{s}^{\mathrm{H}}=1 / 3, \quad 0.6: \pi_{s}^{\mathrm{M}}=1 / 3, \quad 0.3: \pi_{s}^{\mathrm{L}}=1 / 3\right\}, \\
& \mathcal{S}^{\prime}=\left\{0.9: \pi_{s^{\prime}}^{\mathrm{H}}=0.1, \quad 0.6: \pi_{s^{\prime}}^{\mathrm{M}}=0.8, \quad 0.3: \pi_{s^{\prime}}^{\mathrm{L}}=0.1\right\},
\end{aligned}
$$

each of which contains three scenarios (high $(\mathrm{H})$, medium $(\mathrm{M})$, low $(\mathrm{L})$ ) with the corresponding probability of occurrence denoted as $\pi_{s}\left(\pi_{s^{\prime}}\right)$. For both scenario sets, the expected stochastic production is equal to $0.6 \mathrm{p} . \mathrm{u}$. and the reserve requirements to contain every deviation from the expected values are $R R=0.3$ p.u. (both for upward and downward reserves). However, in scenario set $\mathcal{S}^{\prime}$ the expected utilization of reserves is lower than in scenario set $\mathcal{S}$, since the probabilities for deviating from the mean production are only 0.1 instead of $1 / 3$. This difference in expected reserve deployment cost is completely disregarded during the allocation process. 
Finally, a third source of inefficiency relates to network topology issues that are neglected during the allocation process. European reserve and day-ahead energy markets follow a zonal pricing scheme, where each zone is treated as a copper plate and cross-zonal Available Transmission Capacity (ATC) is set with respect to several security measures [38]. The ATC-based allocation is a reasonable approximation if interdependencies between zones are low and market flows are not significantly different than physical flows. However, highly meshed grids usually involve significant loop flows that make impossible to accurately calculate ATCs without complete information about market conditions, especially considering the variable generation patterns of stochastic renewables [27]. This issue will be exacerbated in view of new AC interconnections that will reduce the resistance to physical flows. Nonetheless, the foreseen investments in HVDC lines can efficiently mitigate these issues as long as their controllability is properly incorporated in the market-related operations similar to the transmission allocation process.

\section{Practical modeling and solution approach}

This section describes the three transmission allocation models used in this paper. The structure of these models is presented in Figure 3, where cost functions $\mathcal{C}$ and constraints $\mathcal{Q}$ are augmented by the superscripts $R, D, B$ to denote reserve capacity, day-ahead and balancing markets. Symbol $\mathbb{E}_{s}[\cdot]$ is the expectation operator over the scenario set $\mathcal{S}$.

First, we introduce a set of simplifying but necessary assumptions to obtain a computationally tractable instance of these models. Then, we provide the mathematical formulation of the optimization problems pertaining to the deterministic and stochastic dispatch models as well as the procedure followed to transform the proposed bilevel model into a single-level MILP. Finally, we propose a Benders decomposition approach that exploits the structure of the final MILP formulation and allows to tackle problem instances of realistic size.

\subsection{Modeling assumptions}

Before proceeding to the analytical mathematical formulations of the transmission allocation models, a set of simplifying assumptions must be introduced in order to obtain a solvable instance of these models. Especially for the proposed preemptive transmission allocation model, which is formulated as a bilevel program, it is important to preserve the linear and convex formulation of the lower-level problems that permits to recast the problem as a single-level MILP that can be solved by existing branch-and-bound algorithms.

To this end, we follow the European market paradigm where discrete startup and shut-down costs of conventional generators are internalized in the energy offers assuming also linear cost functions with constant marginal cost. In addition, no inter-temporal constraints, e.g., ramping, are explicitly modeled 

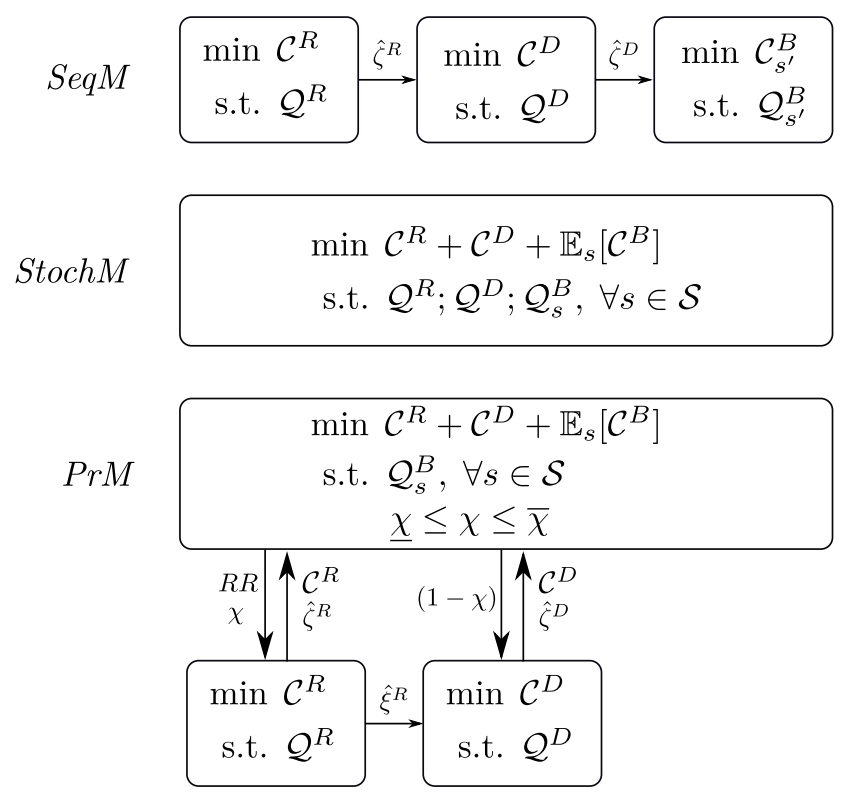

Fig. 3: Schematic representation of transmission allocation models. SeqM: Sequential market clearing. StochM: Stochastic energy and reserves cooptimization. PrM: Preemptive transmission allocation.

under the assumption that each trading floor is settled on independent hourly single-period auctions. Nonetheless, ramping constraints can be incorporated in the proposed framework, extending the formulation to a multi-period setup. These assumptions are in line with the European and in particular the Nordic market design, that operate on a portfolio bidding basis where market players submit hourly offers for their aggregated portfolio instead of unit-specific offers. In addition to single hour portfolio offers, market participants are allowed to submit more complex offers (the so-called 'block orders') that implicitly incorporate generator ramping limits and multi-period cost structures related to on/off status of the units. However, block orders are outside the scope of this paper. The interested reader is referred to [7] for further information on those complex products. It should be noted that for the practical application of the preemptive transmission allocation method, the system operator has to estimate these energy supply curves, since decision making takes place prior to the day-ahead market clearing and thus only reserve capacity offers are actually known. This estimation can be based on historical supply curves or employ more advanced methods to predict energy offers as proposed, e.g., in [53], [39]. The proposed models account for uncertainty from stochastic generation with main focus on wind power. However, other sources of uncertainty such as demand variations can be considered in a similar way.

The AC transmission network is modeled with a lossless DC approximation, where the absolute value of the susceptance of line $\ell$ is denoted as $B_{\ell}$ and $A_{\ell n}$ 
is a line-to-bus incidence matrix whose elements are equal to $-1 / 1$ if line $\ell$ leaves/enters bus $n$ and 0 otherwise. The power flow over a point-to-point HVDC line is modeled as an equal power injection and withdrawal at the receiving and sending ends of the line. In the European context, both reserve and day-ahead markets follow a zonal approach in which intra-zonal network constraints are neglected. The subsequent formulations follow this setup in the reserve capacity market model, but allow for full network representation both in day-ahead and balancing markets. Considering that the proposed model is a decision-support tool for the system operator, it provides the option to account for the impact of domestic grid constraints when deciding for the optimal transmission allocation and regional reserve requirements. Nonetheless, a dayahead zonal setup can be directly incorporated in the model as it practically constitutes a less restrictive version of the current formulation. In this case, the system operator will probably have to bear higher counter-trading costs after day-ahead market clearing to reestablish network feasibility.

\subsection{Transmission capacity allocation models}

This section presents the complete mathematical formulation of the different transmission allocation models. For the sake of conciseness, we start from the sequential model and we explain the necessary reformulations in order to obtain the stochastic and preemptive allocation models.

\subsubsection{Sequential market clearing model (SeqM): Inefficient transmission allocation}

According to current practice, reserve capacity, day-ahead and balancing markets are cleared in sequential and independent auctions. Let $R R_{a}^{+} / R R_{a}^{-}$denote the upward/downward reserve requirements for each area $a \in A$ of the system and $\chi_{e}$ the percentage of inter-area interconnection capacity $T_{e}$ of link $e$ allocated to reserves exchange. Taking these values as input parameters, the reserve capacity market aims at minimizing the total procurement $\operatorname{cost} \mathcal{C}^{R}$ of upward $r_{i a}^{+}$and downward $r_{i a}^{-}$capacity from a pool of common resources $i \in I$ that are available to all system areas. The reserve capacity $\operatorname{costs} C_{i}^{+}$and $C_{i}^{-}$reflect the opportunity cost that generators incur from operating at a setpoint different than their optimal day-ahead energy production. The reserve capacity market clearing is formulated as:

$$
\underset{\Phi R}{\operatorname{Minimize}} \mathcal{C}^{R}=\sum_{a \in A} \sum_{i \in I}\left(C_{i}^{+} r_{i a}^{+}+C_{i}^{-} r_{i a}^{-}\right)
$$

subject to

$$
\begin{aligned}
& \sum_{a} r_{i a}^{+} \leq R_{i}^{+}, \quad \forall i \\
& \sum_{a} r_{i a}^{-} \leq R_{i}^{-}, \quad \forall i
\end{aligned}
$$




$$
\begin{aligned}
& \sum_{i} r_{i a}^{+} \geq R R_{a}^{+}, \quad \forall a, \\
& \sum_{i} r_{i a}^{-} \geq R R_{a}^{-}, \quad \forall a, \\
& \sum_{i \in \mathcal{M}_{a_{s}(e)}^{I}} r_{i a_{r}(e)}^{+} \leq \chi_{e} T_{e}, \quad \forall e, \\
& \sum_{i \in \mathcal{M}_{a_{r}(e)}^{I}} r_{i a_{s}(e)}^{+} \leq \chi_{e} T_{e}, \quad \forall e, \\
& \sum_{i \in \mathcal{M}_{a_{s}(e)}^{I}} r_{i a_{r}(e)}^{-} \leq \chi_{e} T_{e}, \quad \forall e, \\
& \sum_{i \in \mathcal{M}_{a_{r}(e)}^{I}} r_{i a_{s}(e)}^{-} \leq \chi_{e} T_{e}, \quad \forall e, \\
& r_{i a}^{+}, r_{i a}^{-} \geq 0, \quad \forall i, \forall a,
\end{aligned}
$$

where $\Phi^{R}=\left\{r_{i a}^{+}, r_{i a}^{-}, \forall i, \forall a\right\}$ is the set of optimization variables. Constraint (4b) ensures that the provision of upward reserves from unit $i$ to all areas $a$ of the power system does not exceed its upward capacity offer $R_{i}^{+}$. Similarly, constraint (4c) enforces the capacity offer limit $R_{i}^{-}$for downward reserve provision. The upward $R R_{a}^{+}$and downward $R R_{a}^{-}$area reserve requirements are enforced by constraints $(4 \mathrm{~d})$ and $(4 \mathrm{e})$, respectively. The set of constraints (4f)-(4g) models the upper bounds of upward reserves exchange between the sending $a_{s}(e)$ and receiving $a_{r}(e)$ areas of link $e$. The amount of upward reserves $r_{i a}^{+}$procured by area $a$ from unit $i$ which is physically located in area $a^{\prime}$, i.e., subset of units $i \in \mathcal{M}_{a^{\prime}}^{I}$, should not exceed the inter-area exchange limit $\chi_{e} T_{e}$, where $\chi_{e}$ is the percentage of link capacity $T_{e}$ allocated to reserves trade. The same principle applies also for downward reserves exchange limits enforced by constraints (4h)-(4i). In line with the zonal network representation of reserves markets, the transmission capacity $T_{e}$ of link $e$ is the aggregated flow limit of all tie-lines between areas $a_{s}(e)$ and $a_{r}(e)$ given as:

$$
T_{e}=\sum_{\ell \in \Lambda_{a_{r}(e)}^{a_{s}(e)}} T_{\ell}, \quad \forall e,
$$

where $\Lambda_{a_{r}(e)}^{a_{s}(e)}$ is the set of AC and HVDC lines connecting areas $a_{s}(e)$ and $a_{r}(e)$ across link $e$. Finally, constraints $(4 \mathrm{j})$ constitute variable declarations.

Currently the size of reserve requirements in each region of the system is determined by the corresponding operator according to the dimensioning rules defined on the European level [11], [18]. These rules follow a deterministic philosophy that ensures enough reserves to supply a predefined percentage of hourly load and fulfill a set of static reliability criteria, i.e., N-1 security constraint violations. However, this approach disregards the structure of renewables' uncertainty and its impact on operational costs and system reliability. 
Taking into account these considerations, reserve requirements can alternatively be determined using a probabilistic description of system uncertainties that perform a trade-off between risk and reserve cost [14], [37]. The percentage $\chi$ of tie-line capacity that becomes available during the reserve capacity market clearing can be determined according to methods presented in Section 2.3 .

Once the optimal reserve procurement $\left(\hat{r}_{i a}^{-}, \hat{r}_{i a}^{+}\right)$is defined, the day-ahead schedule that minimizes the energy production $\operatorname{costs} \mathcal{C}^{D}$ in $(6 \mathrm{a})$, based on the price offers $C_{i}$ from the conventional generators, is given as the solution to following optimization problem:

$$
\underset{\Phi^{D}}{\operatorname{Minimize}} \mathcal{C}^{D}=\sum_{i \in I} C_{i} p_{i}
$$

subject to

$$
\begin{aligned}
& \sum_{j \in \mathcal{M}_{n}^{J}} w_{j}+\sum_{i \in \mathcal{M}_{n}^{I}} p_{i}-D_{n}- \\
& \sum_{\ell \in L^{\mathrm{AC}}} A_{\ell n} f_{\ell}+\sum_{\ell \in L^{\mathrm{DC}}} A_{\ell n} z_{\ell}=0, \quad \forall n, \\
& \sum_{a} \hat{r}_{i a}^{-} \leq p_{i} \leq P_{i}-\sum_{a} \hat{r}_{i a}^{+}, \quad \forall i, \\
& 0 \leq w_{j} \leq \bar{W}_{j}, \quad \forall j, \\
& f_{\ell}=B_{\ell} \sum_{n} A_{\ell n} \delta_{n}, \quad \forall \ell \in L^{\mathrm{AC}}, \\
& -\left(1-\chi_{\ell}\right) T_{\ell} \leq f_{\ell} \leq\left(1-\chi_{\ell}\right) T_{\ell}, \quad \forall \ell \in L^{\mathrm{AC}}, \\
& -\left(1-\chi_{\ell}\right) T_{\ell} \leq z_{\ell} \leq\left(1-\chi_{\ell}\right) T_{\ell}, \quad \forall \ell \in L^{\mathrm{DC}}, \\
& \delta_{1}=0, \\
& \delta_{n} \text { free, } \forall n ; f_{\ell} \text { free, } \forall \ell \in L^{\mathrm{AC}} ; z_{\ell} \text { free, } \forall \ell \in L^{\mathrm{DC}} .
\end{aligned}
$$

The set of optimization variables is $\Phi^{D}=\left\{p_{i}, \forall i ; w_{j}, \forall j ; \delta_{n}, \forall n ; f_{\ell}, z_{\ell}, \forall \ell\right\}$. Constraint $(6 \mathrm{~b})$ enforces day-ahead power balance at each node $n$, which guarantees that nodal demand $D_{n}$ is equal to the total power injections from conventional units $p_{i}$ and stochastic producers $w_{j}$ located at this node plus the net power flow from the AC and HVDC lines, denoted as $f_{\ell}$ and $z_{\ell}$, respectively. The upper and lower dispatch limits of conventional units are enforced by constraint (6c), taking into account the reserve schedule $\left(\hat{r}_{i a}^{-}, \hat{r}_{i a}^{+}\right)$and the generation capacity $P_{i}$ of each unit $i$. Constraint $(6 \mathrm{~d})$ limits the dispatch of stochastic power production to a point forecast $\bar{W}_{j}$, typically the conditional expectation of the stochastic process. Power flows over the $\mathrm{AC}$ network, given in (6e), are restricted by the day-ahead transmission capacity limits in (6f) considering that only $\left(1-\chi_{\ell}\right)$ percent of the line capacity $T_{\ell}$ is now available for energy trade. Here, inter-area links are disaggregated to represent actual network topology and thus the allocation parameter $\chi_{e}$ is mapped to the line 
components of each link $e$, i.e., $\chi_{e}=\chi_{\ell}, \forall \ell \in \Lambda_{a_{r}(e)}^{a_{s}(e)}$. Similarly, the HVDC line flow limits are encountered by constraint $(6 \mathrm{~g})$. Node 1 is set as the reference in $(6 \mathrm{~h})$ fixing its day-ahead voltage angle to 0 , while the rest of voltage angles as well as the power flows over the AC and HVDC lines are free variables as stated in $(6 \mathrm{i})$.

Moving close to real-time operation when uncertainty is unveiled, i.e., the actual stochastic power production $W_{j s^{\prime}}$ is known, any energy deviations from the day-ahead schedule $\left(\hat{p}_{i}, \hat{w}_{j}, \hat{f}_{\ell}, \hat{z}_{\ell}\right)$ need to be compensated through proper re-dispatch actions. Generation shortages are compensated by the activation of upward regulation $p_{i s^{\prime}}^{+}$or under extreme conditions by resorting to load shedding $l_{n s^{\prime}}^{\text {sh }}$ actions. Similarly, to accommodate generation excess flexible units have to reduce their output to provide down regulation $p_{i s^{\prime}}^{-}$or a portion $w_{j s^{\prime}}^{\text {spill }}$ of stochastic power production has to be spilled.

To determine the re-dispatch actions that minimize balancing $\operatorname{cost} \mathcal{C}^{B}$, the balancing market is cleared based on the optimization model (7). The objective function (7a) to be minimized is the cost of reserve deployment plus the cost of load shedding at a price of $C^{\text {sh }}$ that reflects the value of lost load for the consumers. Considering that the generators awarded to provide reserves have already received a capacity payment, their balancing price offers are equal to their energy marginal cost $C_{i}$.

$$
\underset{\Phi^{B}}{\operatorname{Minimize}} \mathcal{C}^{B}=\sum_{i \in I} C_{i}\left(p_{i s^{\prime}}^{+}-p_{i s^{\prime}}^{-}\right)+\sum_{n \in N} C^{\text {sh }} l_{n s^{\prime}}^{\text {sh }}
$$

subject to

$$
\begin{aligned}
& \sum_{i \in \mathcal{M}_{n}^{I}}\left(p_{i s^{\prime}}^{+}-p_{i s^{\prime}}^{-}\right)+l_{n s^{\prime}}^{\mathrm{sh}}+\sum_{j \in \mathcal{M}_{n}^{J}}\left(W_{j s^{\prime}}-\hat{w}_{j}-w_{j s^{\prime}}^{\mathrm{spill}}\right) \\
& +\sum_{\ell \in L^{\mathrm{AC}}} A_{\ell n}\left(\hat{f}_{\ell}-\tilde{f}_{\ell s^{\prime}}\right)+\sum_{\ell \in L^{\mathrm{DC}}} A_{\ell n}\left(\hat{z}_{A_{\ell n}}-\tilde{z}_{\ell s^{\prime}}\right)=0, \quad \forall n \\
& 0 \leq p_{i s^{\prime}}^{+} \leq \sum_{a} \hat{r}_{i a}^{+}, \quad \forall i, \\
& 0 \leq p_{i s^{\prime}}^{-} \leq \sum_{a} \hat{r}_{i a}^{-}, \quad \forall i \\
& 0 \leq l_{n s^{\prime}}^{\mathrm{sh}} \leq D_{n}, \quad \forall n, \quad, \quad \forall \ell \in L^{\mathrm{AC}} \\
& 0 \leq w_{j s^{\prime}}^{\mathrm{spill}} \leq W_{j s^{\prime}}, \quad \forall j, \\
& \tilde{f}_{\ell s^{\prime}}=B_{\ell} \sum_{n} A_{\ell n} \tilde{\delta}_{n s^{\prime}}, \quad \forall \\
& -T_{\ell} \leq \tilde{f}_{\ell s^{\prime}} \leq T_{\ell}, \quad \forall \ell \in L^{\mathrm{AC}}, \\
& -T_{\ell} \leq \tilde{z}_{\ell s^{\prime}} \leq T_{\ell}, \quad \forall \ell \in L^{\mathrm{DC}}, \\
& \tilde{\delta}_{1 s^{\prime}}=0, \\
& \tilde{\delta}_{1 s^{\prime}} \text { free, } \forall n ; \tilde{f}_{\ell s^{\prime}} \text { free }, \forall \ell \in L^{\mathrm{AC}} ; \quad \tilde{z}_{\ell s^{\prime}} \text { free }, \forall \ell \in L^{\mathrm{DC}}
\end{aligned}
$$


where the set $\Phi^{B}=\left\{p_{i s^{\prime}}^{+}, p_{i s^{\prime}}^{-}, \forall i ; w_{j s^{\prime}}^{\text {spill }}, \forall j ; l_{n s^{\prime}}^{\text {sh }}, \tilde{\delta}_{n s^{\prime}}, \forall n ; \tilde{f}_{\ell s^{\prime}}, \tilde{z}_{\ell s^{\prime}}, \forall \ell\right\}$ contains the optimization variables of model $(7)$. Constraints $(7 \mathrm{c})$ and $(7 \mathrm{~d})$ ensure that upward and downward reserve deployment, $p_{i s^{\prime}}^{+}$and $p_{i s^{\prime}}^{-}$, are within the respective procured amounts from the reserve capacity market. The upper bounds of alternative balancing actions, such as load shedding $l_{n s^{\prime}}^{\text {sh }}$ and stochastic production spillage $w_{j s^{\prime}}^{\text {spill }}$, are set by constraints (7e) and (7f), respectively. Load curtailment cannot be higher than actual consumption, while spilled production is at most equal to the realized stochastic in-feed $W_{j s^{\prime}}$. Real-time AC power flows $\tilde{f}_{\ell s^{\prime}}$ modeled by $(7 \mathrm{~g})$ according to the updated voltage angles $\tilde{\delta}_{n s^{\prime}}$, are limited by the corresponding transmission capacity limits imposed by $(7 \mathrm{~h})$. Likewise, the HVDC flows $\tilde{z}_{\ell s^{\prime}}$ during the balancing operation are bounded by the line capacities in (7i). Equation (7b) ensures that generation, transmission and load are properly re-dispatched to contain the deviation of stochastic generation from the day-ahead stage such that all system nodes remain in balance. In accordance with the day-ahead power flow definition, node 1 is set as the reference by $(7 \mathrm{j})$ and all line flows and remaining voltage angles are declared as free variables by $(7 \mathrm{k})$.

Following this market sequence, the total system cost for the particular realization of stochastic production $W_{s^{\prime}}$ is equal to $\mathcal{C}^{R}+\mathcal{C}^{D}+\mathcal{C}^{B}$. This entails that the minimum operational system cost is determined as the optimal tradeoff of the physically coupled reserve, energy and balancing services. However, the functional decoupling of the corresponding trading floors prevents any temporal coordination and reduces market efficiency as the shares of stochastic generation increase.

\subsubsection{Stochastic energy and reserves co-optimization model (StochM): Perfect transmission allocation}

A straightforward advancement of the existing sequential setup is the cooptimization of reserve capacity and day-ahead energy schedules in order to eliminate the previous market separation pitfall. This joint clearing approach accounts for the complementary and substitutional properties of these two services both in terms of generation and transmission. As a result, the allocation of transmission capacity between energy and reserves is no longer a fixed input, i.e., the parameter $\chi$ of the sequential model, but becomes a byproduct of the clearing process. However, in this co-optimization approach reserve requirements $R R$ remain a deterministic input to the model, while day-ahead decisions are myopic about the cost of actual reserve deployment.

This missing link can be restored if these trading floors are optimized in a probabilistic framework. Using a scenario set $\mathcal{S}$ to model the uncertain in-feed of stochastic producers, with $W_{j s}$ being the realization of stochastic generation of wind farm $j$ in scenario $s$, the stochastic energy and reserves co-optimization model is formulated as the two-stage stochastic programming problem (8). The first-stage constraints $(8 \mathrm{~b})$ pertaining to reserves procurement are derived from model (4), while day-ahead energy constraints (8c)-(8f) are identical to those of model (6). However, given the implicit transmission 
capacity allocation between energy and reserves, constraints (4f)-(4i) become obsolete and the parameter $\chi_{\ell}$ is removed from constraints $(6 \mathrm{f})-(6 \mathrm{~g})$, which are replaced by (8e)-(8f). The second-stage constraints $(8 \mathrm{~g})$ model the recourse actions for all scenarios $s \in \mathcal{S}$, similar to the limits imposed in model (7). As a result, the cost of re-dispatch actions for each scenario $s$ is directly mapped to objective function (8a), weighted by the corresponding probability of occurrence $\pi_{s}$. Having this probabilistic uncertainty representation, day-ahead stochastic production dispatch is not bounded by a single-valued forecast $\bar{W}$ but can take any value up to the installed capacity limit $W$, as shown in (8d).

$$
\begin{aligned}
\underset{\Phi^{S T}}{\operatorname{Minimize}} & \sum_{a \in A} \sum_{i \in I} C_{i}^{+} r_{i a}^{+}+C_{i}^{-} r_{i a}^{-}+C_{i} p_{i} \\
& +\sum_{s \in \mathcal{S}} \pi_{s}\left[\sum_{i \in I} C_{i}\left(p_{i s}^{+}-p_{i s}^{-}\right)+\sum_{n \in N} C^{\mathrm{sh}} l_{n s}^{\mathrm{sh}}\right]
\end{aligned}
$$

subject to

$$
\begin{aligned}
& (4 \mathrm{~b})-(4 \mathrm{e}),(4 \mathrm{j}), \\
& (6 \mathrm{~b})-(6 \mathrm{c}),(6 \mathrm{e}),(6 \mathrm{~h}),(6 \mathrm{i}), \\
& 0 \leq w_{j} \leq W_{j}, \quad \forall j, \\
& -T_{\ell} \leq f_{\ell} \leq T_{\ell}, \quad \forall \ell \in L^{\mathrm{AC}}, \\
& -T_{\ell} \leq z_{\ell} \leq T_{\ell}, \quad \forall \ell \in L^{\mathrm{DC}}, \\
& (7 \mathrm{~b})-(7 \mathrm{k}), \quad \forall s \in \mathcal{S} .
\end{aligned}
$$

The set of optimization variables of model (8) is $\Phi^{S T}=\left\{r_{i a}^{+}, r_{i a}^{-}, \forall i, \forall a ; R R_{a}^{+}\right.$, $R R_{a}^{-}, \forall a ; p_{i}, \forall i ; w_{j}, \forall j ; \delta_{n}, \forall n ; f_{\ell}, z_{\ell}, \forall \ell ; p_{i s}^{+}, p_{i s}^{-}, \forall i, \forall s ; w_{j s}^{\text {spill }}, \forall j, \forall s ; l_{n s}^{\text {sh }}, \tilde{\delta}_{n s}, \forall n$, $\left.\forall s ; \tilde{f}_{\ell s}, \tilde{z}_{\ell s}, \forall l, \forall s\right\}$.

Here, three important remarks are in place. First, notice that reserve requirements $R R$ now become decision variables and thus are endogenously optimized in model (8), in contrast to the sequential model (4) that requires their ex-ante definition. In fact, reserve requirements are redundant decision variables for model (8), given that the stochastic dispatch can pre-position resources even out of merit order to ensure sufficient flexibility during the balancing operation. Nonetheless, keeping this explicit definition of $R R_{a}^{+}, R R_{a}^{-}$ variables, allows to reveal reserve requirements that are optimal in a probabilistic sense. A second important remark is that since reserve and day-ahead energy schedules are co-optimized, transmission capacity is implicitly allocated between these services and the optimal value of $\chi$ can be extracted if necessary by an ex-post calculation. Finally, having a full probabilistic description of stochastic production, the day-ahead dispatch is not bounded by a specific quantile of the predictive distribution, e.g., the conditional mean, but only by the physical limit of installed capacity.

In spite of its favorable properties, the stochastic dispatch model is not compatible with the existing market architecture, since it requires the joint 
optimization of energy and reserve capacity services that is opposed to their separate trading in the European market context. Besides, pricing issues related to cost recovery of flexible producers and revenue adequacy of the market operator remain an open debate in the technical literature [41], [58], [59], since these properties are guaranteed only in expectation but not for every uncertainty realization. Therefore, a real-life implementation of this market model requires that market operators and participants are willing to accept economic losses for some uncertainty outcomes, unless some out-of-the-market payments are made to compensate their economic deficit. Acknowledging that is highly improbable that the stochastic dispatch model is adopted in the short-term future as a market-clearing mechanism, the purpose of this dispatch model in the current work is to define an optimal benchmark for inter-temporal market coordination rather than to advocate for its real-life application.

\subsubsection{Preemptive transmission allocation model (PrM): Bridging the efficiency gap}

Comparing the advanced stochastic dispatch in model (8) with the sequential model (4)-(7), we can deduce that the main source of inefficiency is the ex-ante definition of parameters $R R$ and $\chi$. However, these parameters are controlled solely by the system operator and thus can be used as control parameters to enable the implicit temporal coupling of the reserve capacity, day-ahead and balancing markets. A systematic approach for the optimal definition of these values can be mathematically formulated as the following stochastic bilevel program [56], [51]:

$$
\begin{aligned}
\underset{\Phi^{P R}}{\operatorname{Minimize}} & \sum_{a \in A} \sum_{i \in I} C_{i}^{+} r_{i a}^{+}+C_{i}^{-} r_{i a}^{-}+C_{i} p_{i} \\
& +\sum_{s \in \mathcal{S}} \pi_{s}\left[\sum_{i \in I} C_{i}\left(p_{i s}^{+}-p_{i s}^{-}\right)+\sum_{n \in N} C^{\mathrm{sh}} l_{n s}^{\mathrm{sh}}\right]
\end{aligned}
$$

subject to

$$
\begin{aligned}
& 0 \leq \chi_{e} \leq 1, \quad \forall e, \\
& \text { (7b) }-(7 \mathrm{k}), \quad \forall s \in \mathcal{S} \text {, } \\
& \left(r_{i a}^{+}, r_{i a}^{-}\right) \in \arg \left\{\begin{array}{ll}
\operatorname{Minimize}_{\check{r}_{i a}^{+}, \check{r}_{i a}^{-}} & \sum_{a \in A} \sum_{i \in I}\left(C_{i}^{+} \check{r}_{i a}^{+}+C_{i}^{-} \check{r}_{i a}^{-}\right) \\
\text {subject to } & (4 \mathrm{~b})-(4 \mathrm{j})
\end{array}\right\}, \\
& \left(\begin{array}{c}
p_{i}, w_{j}, \\
\delta_{n}, f_{\ell}, z_{\ell}
\end{array}\right) \in \arg \left\{\begin{array}{ll}
\underset{\check{p}_{i}, \check{w}_{j}, \check{\delta}_{n}, \check{f}_{\ell}, \check{z}_{\ell}}{\operatorname{Minimize}} & \sum_{i \in I} C_{i} \check{p}_{i} \\
\text { subject to } & (6 \mathrm{~b})-(6 \mathrm{i})
\end{array}\right\},
\end{aligned}
$$


where $\Phi^{P R}=\left\{r_{i a}^{+}, r_{i a}^{-}, \forall i, \forall a ; R R_{a}^{+}, R R_{a}^{-}, \forall a ; \chi_{e}, \forall e ; p_{i}, \forall i ; w_{j}, \forall j ; \delta_{n}, \forall n ; f_{\ell}, z_{\ell}\right.$, $\left.\forall \ell ; p_{i s}^{+}, p_{i s}^{-}, \forall i, \forall s ; w_{j s}^{\text {spill }}, \forall j, \forall s ; l_{n s}^{\text {sh }}, \tilde{\delta}_{n s}, \forall n, \forall s ; \tilde{f}_{\ell s}, \tilde{z}_{\ell s}, \forall \ell, \forall s\right\}$ is the set of optimization variables.

The objective function (9a) is identical to (8a) aiming at minimizing the total expected cost of power system operation. The upper-level constraint $(9 \mathrm{~b})$ bounds the decision variable $\chi_{e}$ to the normalized transmission capacity, while constraints $(9 \mathrm{c})$ model the limits of balancing actions for every scenario $s$. The lower level problems (9d) and (9e) reproduce the market clearing of the reserve capacity and day-ahead energy trading floors of the conventional transmission allocation process, according to models (4) and (6), respectively.

In other words, for the same values of parameters $R R$ and $\chi$, the solution of $(9 \mathrm{~d})$ and $(9 \mathrm{e})$ is identical to the one obtained from the sequential clearing by (4) and (6), due to the disjunction of sets $\left\{r_{i a}^{+}, r_{i a}^{-}\right\}$and $\left\{p_{i}, w_{j}, \delta_{n}, f_{\ell}, z_{\ell}\right\}$. Note also that the independence of these sets allows us to model the reserve capacity and day-ahead markets in a single lower-level that actually yields this bilevel formulation instead of a trilevel model. Though, unlike models (4) and (6) where $R R$ and $\chi$ are exogenously defined inputs, these values are now decision variables of the upper-level problem in bilevel model (9). Essentially, this model allows the system operator to decide on the optimal values of $R R$ and $\chi$ anticipating the reaction of reserve capacity and day-ahead markets as well as the resulting expected balancing cost.

The bilevel model (9) can be interpreted as a Stackelberg game [57] in which a particular player (leader) does not only observe but can also anticipate the decisions of other players (followers). Before we proceed to the discussion about model structure, it is important to underline the difference between optimization levels that impose an hierarchical relationship of the players and optimization stages that denote a point in time when new information is revealed. Our model considers an hierarchical relationship in which the system operator acts as leader, while reserve capacity, day-ahead and balancing markets act as followers. In the time domain, the market sequence defines two stages. The first stage comprises reserve capacity and day-ahead markets, that are not co-optimized but both share the same information regarding system uncertainties. Balancing market belongs to the second stage since new information becomes available, i.e., the realization of uncertainty modeled by scenario $s$.

The proposed model (9) differs from the typical bilevel formulation of the Stackelberg game, where upper level pertains only to leader's decisions and lower level models followers' reaction. Here, lower level includes only reserve capacity and day-ahead market-clearing problems (9d) and (9e), respectively, while balancing market is modeled in the upper level by the last term of the objective function (9a) and constraints (9c) for all $s \in \mathcal{S}$. Nonetheless, this structure ensures indeed that the three trading floors are cleared sequentially as well as it enforces the hierarchical relationship between the system operator and market clearing. 
In particular, lower-level problems (9d) and (9e) ensure that reserve capacity and day-ahead costs are serially and independently minimized. The temporal sequence of the two markets in enforced by the problem structure, since upward and downward reserve procurement variables $r_{i a}^{+}, r_{i a}^{-}$can be modified only by (9d) and enter the day-ahead auction (9e) as parameters. In addition, note that neither $(9 \mathrm{~d})$ nor $(9 \mathrm{e})$ has information about the subsequent balancing market. Hence, both auctions are completely myopic about their impact on the real-time re-dispatch costs. Therefore, upper-level constraints (9c) along with the last term of the objective function (9a) reproduce the balancing market clearing of the sequential model (7), independently for each scenario $s$. This is because the outcome of the balancing market cannot induce any changes to the preceding auctions which are 'locked' to the sequential dispatch through $(9 \mathrm{~d})$ and $(9 \mathrm{e})$.

An equivalent formulation of model (9) can be obtained including balancing market also as a lower-level problem. However, this formulation would unnecessarily complicate the model and increase its computational burden. For similar applications of bilevel programming and further discussion on its conceptual aspects, the reader is referred to [50], [41] and [44].

Considering that model (9) serves as decision-support tool for the operator rather than a market-clearing algorithm, decisions regarding $R R$ and $\chi$ can be further parametrized according to the specific requirements of the decision maker, e.g., to account for exogenously imposed regulatory constraints. For instance a minimum level of domestic upward reserves procurement in area $a^{\prime}$ can be modeled in the upper-level as:

$$
\begin{aligned}
& R R_{a^{\prime}}^{+} \geq \underline{R R}_{a^{\prime}}^{+}, \\
& R R_{a^{\prime}}^{-} \geq \underline{R R}_{a^{\prime}}^{-} .
\end{aligned}
$$

Similarly, the operator can impose a cap of $\bar{\chi}_{e}$ on the inter-area transmission capacity of link $e^{\prime}$ allocated to reserves exchange adding the upper-level constraint:

$$
\chi_{e^{\prime}} \leq \bar{\chi}_{e^{\prime}}
$$

Following the discussion of Section 2.2, this preemptive transmission allocation method assumes full coordination among the regional operators during the process of reserve activation. This setup accounts inherently for imbalance netting opportunities between the different regions which have access to a common pool of available resources, ensuring cost-efficient exchange of balancing energy. In terms of coordination on the procurement phase, the proposed model can be easily adapted to different degrees of coordination. Having reserve requirements as a decision variable of the upper-level problem accomplishes an implicit reserve sharing policy, since this mechanism can be interpreted as a perfectly coordinated dimensioning of reserves. On the other hand, enforcing fixed regional reserve requirements implies a reserve exchange setup that provides access to cheap cross-border resources, but does not prevent the overestimation of reserve requirements. 
In order to harness the full benefits of the stochastic optimization framework, uncertain electricity production is modeled by a finite set $\mathcal{S}$ of scenarios that captures the spatial structure of the forecast errors. This requires from the system operator to obtain this probabilistic information for different geographical locations. This requirement is in line with the reserve dimensioning processes considered by the European system operators based on probabilistic forecasts [31]. In these reserve quantification methods, system-wide probabilistic predictions are centrally obtained by the system operators without information exchange with the market participants.

Our preemptive allocation model, being a decision-support tool for the system operator, uses statistical scenarios to model uncertain in-feed. However, this data should not be directly provided by the stochastic producers or must be openly disseminated to all market participants. Hence, we do respect current market rules and allow stochastic producers to maintain their offering strategies based on single-valued quantity offers. This allows overcoming a major practical caveat of the stochastic dispatch in which all players have to accept settlements based on a common scenario set. In fact, the proposed model 'masks' this probabilistic view of the system operator regarding renewable generation forecast uncertainty even in the existing deterministic market setup.

Considering the proposed formulation (9) one can note that this bilevel model is not computationally tractable given the nested optimization problems (9d) and (9e) that appear in the lower level. However, for a fixed value of the upper-level decision variables $\chi_{e}, R R_{a}^{+}, R R_{a}^{-}$, these optimization problems are linear and thus can be replaced by their Karush-Kuhn- Tucker (KKT) conditions. Before describing this transformation, for notation brevity, we compactly write (9d) as:

$$
\underset{\tilde{\mathbf{x}}^{\mathbf{r}}}{\operatorname{Minimize}} \mathbf{c}_{\mathbf{r}}^{\top} \check{\mathbf{x}}^{\mathbf{r}}
$$

subject to

$$
\begin{aligned}
& Q_{\mathbf{r}} \check{\mathbf{x}}^{\mathbf{r}} \geq q_{\mathbf{r}}: \nu_{\mathbf{r}}, \\
& \mathbf{H}_{\mathbf{r}} \check{\mathrm{x}}^{\mathbf{r}} \geq \chi^{\top} \mathbf{T}: \xi_{\mathbf{r}},
\end{aligned}
$$

where the vectors $\check{\mathbf{x}}^{\mathbf{r}}$ and $\mathbf{c}_{\mathbf{r}}$ are properly stacked versions of decision variables $r_{i a}^{-}, r_{i a}^{+}$and the corresponding reserve capacity costs $C_{i}^{-}, C_{i}^{+}$, respectively. Constraint (12b) includes (4b)-(4e), while (12c) groups constraints (4f) -(4i). The corresponding dual variables are indicated after each constraint following a colon. Then, the KKT conditions of (12) are:

$$
\begin{aligned}
& \mathbf{c}_{\mathbf{r}}-\mathbf{H}_{\mathbf{r}} \boldsymbol{\xi}_{\mathbf{r}}-\mathbf{Q}_{\mathbf{r}} \nu_{\mathbf{r}}=0, \\
& 0 \leq \mathbf{H}_{\mathbf{r}} \check{\mathbf{x}}^{\mathbf{r}}-\chi^{\top} \mathbf{T} \perp \boldsymbol{\xi}_{\mathbf{r}} \geq 0, \\
& 0 \leq \mathbf{Q}_{\mathbf{r}} \check{\mathbf{x}}^{\mathbf{r}}-\mathbf{q}_{\mathbf{r}} \perp \boldsymbol{\nu}_{\mathbf{r}} \geq 0,
\end{aligned}
$$

where operator $\perp$ indicates that the product of the expressions on its right and left side is equal to zero. Constraint (13a) states the stationarity conditions, while (13b) and (13c) are complementary slackness conditions. 
Similarly, indicating by $\check{\mathbf{x}}^{\mathbf{d}}$ the decision variables of the lower-level problem (9e) and $\mathbf{c}_{\mathbf{d}}$ the respective cost coefficients, the day-ahead market clearing is written in a compact form as:

$$
\underset{\check{\mathbf{x}}^{\mathbf{d}}}{\operatorname{Minimize}} \mathbf{c}_{\mathbf{d}^{\top}} \check{\mathbf{x}}^{\mathbf{d}}
$$

subject to

$$
\begin{aligned}
& B_{d} \check{\mathbf{x}}^{\mathbf{d}}=\mathbf{d}: \lambda^{\mathbf{d}}, \\
& \mathbf{Q}_{\mathbf{d}} \check{\mathbf{x}}^{\mathbf{d}} \geq \mathbf{M}_{\mathbf{d}} \mathbf{x}^{\mathbf{r}}+\mathbf{q}_{\mathbf{d}}: \nu_{\mathbf{d}}, \\
& \mathbf{H}_{\mathbf{d}} \check{\mathbf{x}}^{\mathrm{d}} \geq(\mathbf{I}-\chi)^{\top} \mathbf{T}: \xi_{\mathbf{d}},
\end{aligned}
$$

where equation (14b) includes the power balance constraint (6b) and the reference node declaration (6h). Generation-side constraints (6c)-(6d) are aggregated in $(14 \mathrm{c})$ and transmission-side limits $(6 \mathrm{e})-(6 \mathrm{~g})$ are grouped in $(14 \mathrm{~d})$. Then, model (14) can be replaced by its equivalent KKT conditions:

$$
\begin{aligned}
& \mathbf{c}_{\mathbf{d}}-\mathbf{H}_{\mathbf{d}} \boldsymbol{\xi}_{\mathbf{d}}-\mathbf{Q}_{\mathbf{d}} \nu_{\mathbf{d}}=0, \\
& \mathbf{B}_{\mathbf{d}} \check{\mathbf{x}}^{\mathbf{d}}=\mathbf{d}, \lambda^{\mathbf{d}} \quad \text { free, } \\
& 0 \leq \mathbf{H}_{\mathbf{d}} \check{\mathbf{x}}^{\mathbf{d}}-(\mathbf{I}-\chi)^{\top} \mathbf{T} \perp \boldsymbol{\xi}_{\mathbf{d}} \geq 0, \\
& 0 \leq \mathbf{Q}_{\mathbf{d}} \check{\mathbf{x}}^{\mathbf{d}}-\mathbf{M}_{\mathbf{d}} \mathbf{x}^{\mathbf{r}}+\mathbf{q}_{\mathbf{d}} \perp \boldsymbol{\nu}_{\mathbf{d}} \geq 0 .
\end{aligned}
$$

Stationarity conditions are included in (15a), while constraint (15b) along with the left-hand side of complementarity slackness conditions (15c)-(15d) define the primal feasibility space. The complete set of KKT conditions of lower-level problems (9d) and (9e) is provided in Appendix A.

After the above transformations, the initial bilevel problem (9) is reformulated as a single-level MPEC with the following structure:

$$
\begin{aligned}
\underset{\Phi^{P R}}{\operatorname{Minimize}} & \sum_{a \in A} \sum_{i \in I} C_{i}^{+} r_{i a}^{+}+C_{i}^{-} r_{i a}^{-}+C_{i} p_{i} \\
& +\sum_{s \in \mathcal{S}} \pi_{s}\left[\sum_{i \in I} C_{i}\left(p_{i s}^{+}-p_{i s}^{-}\right)+\sum_{n \in N} C^{\mathrm{sh}} l_{n s}^{\mathrm{sh}}\right]
\end{aligned}
$$

subject to

$$
\begin{aligned}
& 0 \leq \chi_{e} \leq 1, \quad \forall e, \\
& (13 \mathrm{a})-(13 \mathrm{c}), \\
& (15 \mathrm{a})-(15 \mathrm{~d}), \\
& (7 \mathrm{~b})-(7 \mathrm{k}), \quad \forall s \in \mathcal{S} .
\end{aligned}
$$

Note that the single-level model (16) is actually a two-stage stochastic programming problem. The first-stage constraints are the KKT conditions of the lower-level problems (16c) and (16d) of the initial bilevel formulation (9) as well as the upper-level constraint pertaining to transmission allocation (16b). 
Given that the separation of reserve capacity and day-ahead markets is fictitious in terms of information disclosure, both trading floors correspond essentially to the same stage despite their sequential clearing that is still guaranteed by the sets of KKT conditions (16c) and (16d). The second-stage constraints (16e) comprise the real-time power balance and the limits of balancing actions for every scenario $s \in \mathcal{S}$, while the corresponding expected costs are included in the objective function (16a).

\subsection{Solution algorithm}

Considering the particular structure of the single-level MPEC (16) we can notice that for a fixed schedule of reserve capacity and day-ahead markets, the second-stage constraints (16e) are independent per scenario. Taking into account that these constraints are linear and thus convex with respect to firststage decisions, allows us to efficiently solve this problem using a Benders decomposition scheme [10].

The Benders master problem in iteration $\eta$ is formulated as:

$$
\underset{\Phi^{B M}}{\operatorname{Minimize}} \sum_{a \in A} \sum_{i \in I} C_{i}^{+} r_{i a}^{+}+C_{i}^{-} r_{i a}^{-}+C_{i} p_{i}+\sum_{s \in \mathcal{S}} \pi_{s} \theta_{s}
$$

subject to

$$
\begin{aligned}
\theta_{s} & \geq \theta^{\mathrm{min}} \\
\theta_{s} & \geq \mathcal{C}_{s}^{B ;(m)} \\
& +\sum_{i} \sum_{a} \gamma_{i a s}^{r^{+} ;(m)}\left(r_{i a}^{+}-r_{i a}^{+;(m)}\right)+\gamma_{i a s}^{r^{-} ;(m)}\left(r_{i a}^{-}-r_{i a}^{-;(m)}\right) \\
& +\sum_{j} \gamma_{j s}^{w ;(m)}\left(w_{j}-w_{j}^{(m)}\right)+\sum_{n} \gamma_{n s}^{\delta ;(m)}\left(\delta_{n}-\delta_{n}^{(m)}\right) \\
& +\sum_{\ell} \gamma_{\ell s}^{f ;(m)}\left(f_{\ell}-f_{\ell}^{(m)}\right)+\gamma_{\ell s}^{z ;(m)}\left(z_{\ell}-z_{\ell}^{(m)}\right), \forall m=1, \ldots, \eta-1, \\
& (16 \mathrm{~b})-(16 \mathrm{~d}),
\end{aligned}
$$

where $\Phi^{B M}=\left\{r_{i a}^{+}, r_{i a}^{-}, \forall i, \forall a ; R R_{a}^{+}, R R_{a}^{-}, \forall a ; \chi_{e}, \forall e ; p_{i}, \forall i ; w_{j}, \forall j ; \delta_{n}, \forall n ; f_{\ell}, z_{\ell}\right.$, $\left.\forall \ell ; \theta_{s}, \forall s\right\}$ is the set of optimization variables of the Benders master problem.

This is a multi-cut version of the Benders decomposition algorithm, where in every iteration $m$ a new set of cuts (17c), one for each scenario $s$, are added in the master problem. These cuts are supporting hyperplanes of a function that maps first-stage decisions to the best possible outcome of the second stage using the auxiliary variable $\theta_{s}$, which is lower bounded by the parameter $\theta^{\mathrm{min}}$. This method relies on the Lagrange multipliers $\gamma$ of the second-stage subproblems that are formulated for each scenario $s \in \mathcal{S}$, as follows: 


$$
\underset{\Phi_{s^{\prime}}^{B, S}}{\operatorname{Minimize}} \mathcal{C}_{s^{\prime}}^{B ;(m)}=\sum_{i \in I} C_{i}\left(p_{i s^{\prime}}^{+}-p_{i s^{\prime}}^{-}\right)+\sum_{n \in N} C^{\mathrm{sh}} l_{n s^{\prime}}^{\mathrm{sh}}
$$

subject to

$$
\begin{aligned}
& r_{i a}^{+}=r_{i a}^{+;(m)}: \gamma_{i a s}^{r^{+} ;(m)}, \quad \forall i, \forall a, \\
& r_{i a}^{-}=r_{i a}^{-;(m)}: \gamma_{i a s}^{r^{-} ;(m)}, \quad \forall i, \forall a, \\
& w_{j}=w_{j}^{(m)}: \gamma_{j s}^{w ;(m)}, \quad \forall j, \\
& \delta_{n}=\delta_{n}^{(m)}: \gamma_{n s}^{\delta ;(m)}, \quad \forall n, \\
& f_{\ell}=f_{\ell}^{(m)}: \gamma_{\ell s}^{f ;(m)}, \quad \forall \ell, \\
& z_{\ell}=z_{\ell}^{(m)}: \gamma_{\ell s}^{z ;(m)}, \quad \forall \ell, \\
& (7 \mathrm{~b})-(7 \mathrm{k}), \quad s=s^{\prime},
\end{aligned}
$$

where $\Phi_{s^{\prime}}^{B S}=\left\{r_{i a}^{+}, r_{i a}^{-}, \forall i, \forall a ; R R_{a}^{+}, R R_{a}^{-}, \forall a ; p_{i}, \forall i ; w_{j}, \forall j ; \delta_{n}, \forall n ; f_{\ell}, z_{\ell}, \forall \ell ; p_{i s^{\prime}}^{+}\right.$, $\left.p_{i s^{\prime}}^{-}, \forall i ; w_{j s^{\prime}}^{\text {spill }}, \forall j ; l_{n s^{\prime}}^{\text {sh }}, \tilde{\delta}_{n s^{\prime}}, \forall n ; \tilde{f}_{\ell s}, \tilde{z}_{\ell s^{\prime}}, \forall \ell\right\}$ is the set of optimization variables of the Benders subproblem for scenario $s^{\prime}$. Constraints (18b)-(18g) fix the complicating variables to the values obtained from the last solution of the master problem. The dual variables $\gamma$ associated with these constraints are practically equal to the sum of the Lagrangian multipliers of the second-stage constraints (18h) in which each complicating variable appears. Allowing for load shedding and wind spillage recourse actions, subproblems (18) are always feasible and thus no feasibility cuts are needed in the master problem (17).

Here, it can be noted that the current formulation of the master problem (17) is non-convex due to the nonlinear complementarity constraints (13a)(13c), (15c)-(15d) that require the product of two linear expressions be equal to 0 . However, these conditions can be linearized by employing SOS1 variables. For example, introducing a non-negative auxiliary variable $\mathbf{u}$ according to (19a) and (19b), constraint (13b) is written as:

$$
\begin{aligned}
& \mathbf{u}=\mathbf{H}_{\mathbf{r}} \check{\mathbf{x}}^{\mathbf{r}}-\chi^{\top} \mathbf{T} \\
& \mathbf{u} \geq 0 \\
& \boldsymbol{\xi}_{\mathbf{r}} \geq 0 \\
& \operatorname{SOS} 1\left(\boldsymbol{\xi}_{\mathbf{r}}, \mathbf{u}\right)
\end{aligned}
$$

where $(19 \mathrm{~d})$ defines an SOS1 relation between $\xi_{\boldsymbol{r}}$ and $\mathbf{u}$ stating that only one of those variables can be non-zero at the optimal point. Constraint (19c) is equivalent to the right-hand side of (13b). Applying this reformulation to all complementarity constraints, the master problem (17) can be solved with standard branch-and-bound solvers that can branch directly on the SOS1 order information or translate them into a formulation with binary variables that yields a MILP instance. 
Finally, it should be pointed out that the linearization of complementarity constraints using SOS1 variables is only one of the different approaches proposed in the literature for solving MPECs. An alternative technique is the Fortuny-Amat transformation [23] that employs a set of disjunctive constraints to linearize expressions of the form $y^{\top} g(x, y), y \geq 0, g(x, y) \geq 0$, using a binary variable and a large constant $K$. Despite widely used in MPEC applications, one practical challenge of Fortuny-Amat transformation is the proper selection of $K$ that may have significant impact on the computational performance of the model. Using another approach, complementarity constraints can be replaced by the strong duality condition that requires primal and dual objective functions to be equal at the optimum. However, in our case the strong duality condition is a nonlinear constraint and thus there is no guarantee that we can obtain the global optimal solution. We refer the interested reader to [24] and [49] for a more detailed presentation of the MPEC framework and of alternative solution techniques.

\section{Results and discussion}

In this section, we first use an illustrative six-bus system to provide and discuss some intuitive solutions of the transmission allocation models described in the previous sections. Then, these models are applied in a realistic case study.

\subsection{Illustrative example}

This section illustrates the features of the three transmission allocation models described in Section 3 using an illustrative case study. We consider the six-bus power system depicted in Figure 4 which comprises two areas. The intraarea transmission network in both areas consists of $\mathrm{AC}$ lines with capacity and reactance equal to $100 \mathrm{MW}$ and 0.13 p.u., respectively. The two tie-lines between areas 1 and 2 are HVDC links with capacity of 20 MW each.

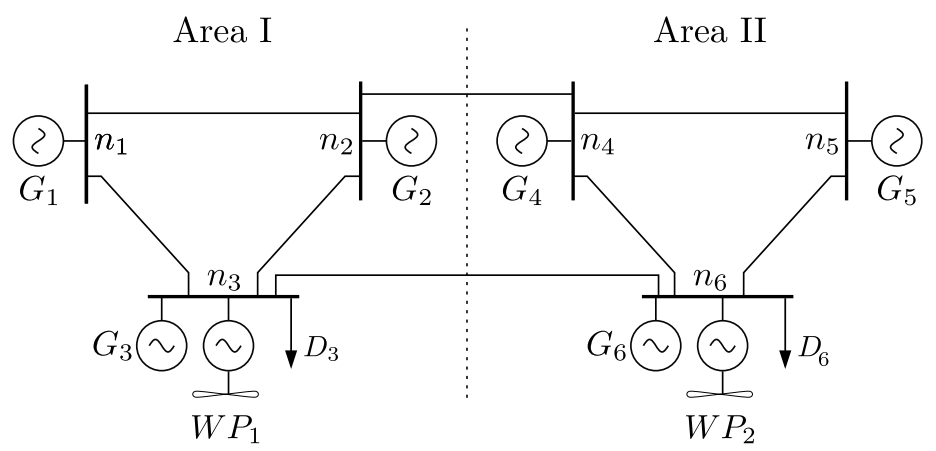

Fig. 4: Six-node interconnected power system. 
Table 2: Generator data.

\begin{tabular}{lcccccc}
\hline \multicolumn{1}{c}{ Unit } & $G_{1}$ & $G_{2}$ & $G_{3}$ & $G_{4}$ & $G_{5}$ & $G_{6}$ \\
\hline$C($ (\$MWh $)$ & 20 & 30 & 40 & 25 & 35 & 45 \\
$P(\mathrm{MW})$ & 120 & 50 & 50 & 120 & 50 & 50 \\
\hline
\end{tabular}

Table 3: Wind power scenarios (in p.u. of installed capacity).

\begin{tabular}{lcc}
\hline Wind power plant & $W P_{1}$ & $W P_{2}$ \\
\hline Scenario $s_{1}$ & 1 & 0.3 \\
Scenario $s_{2}$ & 0.25 & 1 \\
\hline
\end{tabular}

The day-ahead price offers $C$ and the generation capacity $P$ of conventional units are provided in Table 2. Units $G_{1}$ and $G_{4}$ are inflexible, while all remaining units offer half of their capacity for upward and downward reserves provision at cost equal to $10 \%$ of their day-ahead energy offer $C$. The cost of load shedding $C^{\text {sh }}$ is equal to $\$ 1000 / \mathrm{MWh}$ for the inelastic electricity demand $\left(D_{3}\right.$ and $\left.D_{6}\right)$ of 220 and $190 \mathrm{MW}$ at nodes 3 and 6 , respectively. In addition, two wind power plants, $W P_{1}$ and $W P_{2}$, with installed capacity of 50 and 110 MW, respectively, are located at nodes 3 and 6 . The stochastic wind power generation is modeled using two scenarios, $s_{1}$ and $s_{2}$, listed in Table 3 with probability of occurrence 0.6 and 0.4 , respectively. Hence, the corresponding expected wind power production for $W P_{1}$ is equal to 35 and $63.8 \mathrm{MW}$ for $W P_{2}$. Wind power price offers and subsequently the wind power spillage cost are considered to be zero.

According to the business-as-usual market design, the sequential model $(\operatorname{SeqM})$ is based on the independent clearing of the reserve capacity (RC), day-ahead (DA) and balancing (BA) trading floors. Following the prevailing approach in which regional capacity markets are cleared separately, i.e., there is no sharing of cross-border reserve capacity resources, we set the percentage of inter-regional interconnection capacity allocated to reserves exchange equal to $\chi=0$. In that case, reserve requirements for each area are calculated based on the available probabilistic forecasts, such that the larger negative and positive deviations from the expected wind power production, foreseen in the scenario set are covered by domestic resources. For example, the upward $\left(R R^{+}\right)$and downward $\left(R R^{-}\right)$area reserve requirements for area 1 are calculated as:

$$
\begin{aligned}
& R R^{+}=\bar{W}_{1}-\min \left\{s_{1}, s_{2}\right\} \cdot W_{1}=35-12.5=22.5 \mathrm{MW}, \\
& R R^{-}=\max \left\{s_{1}, s_{2}\right\} \cdot W_{1}-\bar{W}_{1}=50-35=15 \mathrm{MW},
\end{aligned}
$$

where $\bar{W}_{1}$ and $W_{1}$ denote the expected production and installed capacity of wind power plant $W P_{1}$, respectively. These values are summarized along with the corresponding reserve requirements for area 2 in Table 4 . 
Table 4: Area reserve requirements (values in MW).

\begin{tabular}{lcc}
\hline $\begin{array}{l}\text { Reserve } \\
\text { Requirements }\end{array}$ & $R R^{+}$ & $R R^{-}$ \\
\hline Area 1 & 22.5 & 15 \\
Area 2 & 30.8 & 46.2 \\
\hline
\end{tabular}

Based on the above information, the market outcomes for the $S e q M$ model are presented in Table 5 for each trading floor and the corresponding costs are provided in Table 6 . The resulting power flows over the system are provided in Appendix B - Table 11. First, we can notice that due to the segmented reserve capacity market, the procured amount of reserves in each area is equal to the corresponding reserve requirements. Having these values as fixed input, the day-ahead market is cleared on the basis of the least-cost merit-order principle accounting both for the intra- and inter-area network constraints. As a result, the inflexible but cheap units $G_{1}$ and $G_{4}$ are dispatched at full capacity, while the day-ahead schedule of the two wind farms is equal to the corresponding expected production. The provision of downward reserves from units $G_{5}$ and $G_{6}$ enforces their day-ahead dispatch, while the remaining electricity demand is covered by unit $G_{2}$. This day-ahead generation schedule results in a power flow of $40 \mathrm{MW}$ from area 2 to area 1, i.e., $20 \mathrm{MW}$ of energy are transfered through each HVDC tie-line. It should be noted that for the current system/market setup this is actually the optimal value of inter-regional trade, i.e., any additional tie-line capacity would remain idle.

Table 5: Market outcomes for the SeqM and StochM transmission allocation models (values in MW).

\begin{tabular}{|c|c|c|c|c|c|c|c|c|c|c|}
\hline \multirow{3}{*}{$\begin{array}{l}\text { Model } \\
\text { Agent }\end{array}$} & \multicolumn{5}{|c|}{ SeqM } & \multicolumn{5}{|c|}{ StochM } \\
\hline & \multicolumn{2}{|c|}{$\mathrm{RC}$} & $\mathrm{DA}$ & \multicolumn{2}{|c|}{$\mathrm{BA}$} & \multicolumn{2}{|c|}{$\mathrm{RC}$} & \multirow[t]{2}{*}{ DA } & \multicolumn{2}{|l|}{$\mathrm{BA}$} \\
\hline & $R R^{-}$ & $R R^{+}$ & & $s_{1}$ & $s_{2}$ & $R R^{-}$ & $R R^{+}$ & & $s_{1}$ & $s_{2}$ \\
\hline$G_{1}$ & 0 & 0 & 120 & 0 & 0 & 0 & 0 & 120 & 0 & 0 \\
\hline$G_{2}$ & 15 & 22.5 & 25 & +22.5 & +15 & 0 & 10 & 40 & +10 & 0 \\
\hline$G_{3}$ & 0 & 0 & 0 & 0 & 0 & 0 & 0 & 7.5 & 0 & 0 \\
\hline$G_{4}$ & 0 & 0 & 120 & 0 & 0 & 0 & 0 & 120 & 0 & 0 \\
\hline$G_{5}$ & 25 & 25 & 25 & +14.5 & -25 & 4.5 & 25 & 25 & +25 & -4.5 \\
\hline$G_{6}$ & 21.2 & 5.8 & 21.2 & -21.2 & -21.2 & 0 & 0 & 0 & 0 & 0 \\
\hline$W P_{1}$ & - & - & 35 & +15 & -22.5 & - & - & 50 & 0 & -37.5 \\
\hline$W P_{2}$ & - & - & 63.8 & -30.8 & +46.2 & - & - & 68 & -35 & +42 \\
\hline
\end{tabular}

Table 6: Comparison of expected system cost (values in $\$$ ).

\begin{tabular}{lcccc}
\hline Model & RC & DA & BA & Total \\
\hline SeqM & 409.0 & 7979.0 & 2585.5 & 10973.5 \\
StochM & 133.3 & 7775.5 & 642.0 & 7832.8 \\
PrM1 & 396.5 & 7954.0 & -262.0 & 8088.5 \\
PrM2 & 208.3 & 7679.5 & 20.0 & 7907.8 \\
\hline
\end{tabular}


Moving to real-time operation, any imbalances resulting from the actual wind power realization being different than the day-ahead schedule need to be compensated by proper re-dispatch actions. In particular, if scenario $s_{1}$ realizes, the net system imbalance is $-15.8 \mathrm{MW}$ which is covered deploying upward reserves. On the other hand, if scenario $s_{2}$ realizes, area 1 faces a production shortage of $22.5 \mathrm{MW}$ and area 2 a production surplus of 46.2 MW. In this case, the congested HVDC tie-lines do not allow for imbalance netting, while the congestion of the domestic AC network of area 1 permits to deploy only up to 15 out of $22.5 \mathrm{MW}$ of available upward reserves from unit $G_{2}$. As a result, the remaining $22.5-15=7.5 \mathrm{MW}$ are covered from costly load shedding at node $3\left(l_{n_{3}, s_{2}}^{\text {sh }}=7.5 \mathrm{MW}\right)$. This situation highlights the inefficiency of the current transmission allocation approach in terms of neglecting network topology, as described in Section 2.4, and underlines the need to account for grid limitations on the actual deployment of reserves during the reserve quantification process.

Another interesting outcome of $S e q M$ model is that in scenario $s_{1}$ unit $G_{6}$ is dispatched for down-regulation exacerbating the imbalance of the system, which in turn has to be covered by the additional up-regulation from units $G_{2}$ and $G_{5}$. At first glance this is a counter-intuitive market outcome which underlines though the inefficiency of a segmented reserve capacity market. Due to restricted inter-area trade, units with high energy cost are assigned to provide downward reserves violating the day-ahead merit order. This inefficient dispatch is partially fixed by down-regulating unit $G_{6}$ in order to replace it by cheaper production from units $G_{2}$ and $G_{5}$ that otherwise would remain idle.

In order to obtain a lower bound of the expected system cost, we employ the stochastic energy and reserves co-optimization model (StochM) that enables the perfect temporal integration of the three considered trading floors. According to Table 6 , model Stoch $M$ yields an expected system cost of $\$ 7832.8$, which translates to $28 \%$ reduction compared to the cost of $\$ 10973.5$ from SeqM model. Having complete information about the spatial characteristics of wind power uncertainty, the stochastic model can endogenously compute the optimal reserve requirements in each area and properly allocate transmission capacity between energy and reserve services.

Exploiting the ability to dispatch wind power in a different value than its expected production, as shown in Table 5, Stoch $M$ model reduces the amount of reserves and schedules flexible generation anticipating potential real-time grid congestions that would prevent the deployment of reserves. Another difference from the $S e q M$ model is that day-ahead power flows on the two HVDC tie-lines have opposite direction, i.e., $20 \mathrm{MW}$ are transferred from area 2 to area 1 through the HVDC link $\left(n_{2}, n_{4}\right)$, while $17.5 \mathrm{MW}$ are exported from area 1 through the HVDC link $\left(n_{3}, n_{6}\right)$. It should be noted that StochM solution does not cause any load shedding actions. However, this solution cannot be directly applied to the SeqM model, since the existing market structure treats all interconnections as a single link by neglecting intra-area network constraints at the reserve capacity market stage. Day-ahead and real-time power flows for the StochM model are provided in Appendix B - Table 11. 
Taking into account these market design limitations, the system operator has to find the optimal value of the parameter $\chi$ and the reserve requirements in each area that minimize the expected system cost. Using a version of the preemptive transmission allocation model that is aligned with the reserves exchange policy, we compute the percentage $\chi$ of the inter-area HVDC capacity that should be allocated for reserves exchanges. The results of this preemptive model (PrM1) are presented in Table 7, where the values of $R R^{-}$and $R R^{+}$shown in parenthesis indicate the amount of reserves destined for meeting the requirements of the neighboring area. The corresponding power flows are shown in Appendix B - Table 12. Despite having the same area reserve requirements as the sequential $S e q M$ model, the expected system cost is reduced to $\$ 8088.5$ (see Table 6 ) by allowing $\chi^{*}=12.5 \%$ of the inter-area transmission capacity to be used for reserves exchange.

Table 7: Market outcomes for the PrM1 and PrM2 transmission allocation models (values in MW).

\begin{tabular}{|c|c|c|c|c|c|c|c|c|c|c|}
\hline Model & \multicolumn{5}{|c|}{ PrM1 } & \multicolumn{5}{|c|}{ PrM2 } \\
\hline \multirow{2}{*}{ Agent } & \multicolumn{2}{|c|}{$\mathrm{RC}$} & $\mathrm{DA}$ & \multicolumn{2}{|c|}{ BA } & \multicolumn{2}{|c|}{$\mathrm{RC}$} & $\mathrm{DA}$ & \multicolumn{2}{|c|}{$\mathrm{BA}$} \\
\hline & $R R^{-}$ & $R R^{+}$ & & $s_{1}$ & $s_{2}$ & $R R^{-}$ & $R R^{+}$ & & $s_{1}$ & $s_{2}$ \\
\hline$G_{1}$ & 0 & 0 & 120 & 0 & 0 & 0 & 0 & 120 & 0 & 0 \\
\hline$G_{2}$ & $15(5)$ & $20(5)$ & 25 & +25 & +15 & 25 & 0 & 50 & 0 & -10 \\
\hline$G_{3}$ & 0 & 2.5 & 5 & 0 & +2.5 & 7.5 & 0 & 7.5 & 0 & 0 \\
\hline$G_{4}$ & 0 & 0 & 120 & 0 & 0 & 0 & 0 & 120 & 0 & 0 \\
\hline$G_{5}$ & 25 & 25 & 25 & +7 & -25 & 13.7 & 15.8 & 13.7 & +15.8 & -13.7 \\
\hline$G_{6}$ & 16.2 & 0.8 & 16.2 & -16.2 & -16.2 & 0 & 0 & 0 & 0 & 0 \\
\hline$W P_{1}$ & 0 & 0 & 35 & +15 & -22.5 & 0 & 0 & 35 & +15 & -22.5 \\
\hline$W P_{2}$ & 0 & 0 & 63.8 & -30.8 & +46.2 & 0 & 0 & 63.8 & -30.8 & +46.2 \\
\hline
\end{tabular}

This transmission allocation provides access to cheaper reserve capacity for area 2, shifting an amount of reserves from the expensive unit $G_{6}$ to the cheaper unit $G_{2}$ and enforcing the provision of $2.5 \mathrm{MW}$ of upward reserves from unit $G_{3}$. Furthermore, this reserve procurement schedule re-adjusts the day-ahead merit order such that unit $G_{3}$ is now dispatched to $5 \mathrm{MW}$. As a result, if scenario $s_{2}$ realizes, area 1 can efficiently contain wind power imbalance by deploying local upward reserves (units $G_{2}$ and $G_{3}$ ) and exploiting the flexibility of HVDC tie-lines that have enough remaining capacity to 'import' up-regulation from area 2. In that case, the 'smart' tuning of the transmission allocation parameter $\chi$ eliminates the need for load curtailment and brings expected system cost closer to the stochastic ideal solution. Note that the uncoordinated quantification of reserves for the two areas still leads to over-procurement of downward reserve capacity from the expensive unit $G_{6}$. As explained in Section 2.4, existing transmission allocation methods do not account for the complementary and substitutional relationship of energy and reserves. On the contrary, model PrM1 takes into account the trade-off between these services to optimally allocate them among the different units, despite that area reserve requirements are fixed to the values of SeqM model. 
Aiming to improve the spatial coordination of reserve capacity markets, regional system operators can cooperate during the reserve quantification process in order to leverage the smoothing effects of stochastic wind power generation. To this end, an enhanced version of the preemptive transmission allocation model, denoted as PrM2, is formulated according to model (9), including as decision variables the area reserve requirements $R R$ in addition to the inter-area transmission capacity $\chi$ allocated to reserves exchange. The results of model PrM2 are presented in Table 7 and the corresponding costs are included in Table 6. The corresponding power flows are provided in Appendix B - Table 12. Having as extra degree of freedom the area reserve requirements, the expected system cost is further decreased to $\$ 7907.8$, indicating that the caveats of the current market structure can be mitigated through the proper setting of certain parameters that are controlled by the system operator. Similar to PrM1 model, this solution prevents load shedding for all scenarios.

Following a similar philosophy as the stochastic approach, the upper level of model PrM2 finds the optimal reserve requirements anticipating the reaction of the all trading floors and their inherent restrictions, e.g., wind power day-ahead dispatch equal to the expected production. Based on this decisionsupport tool, reserve requirements are reduced compared to the sequential model (see Table 8) and the optimal value of transmission allocation is now $\chi^{*}=0 \%$. An interesting observation thought is that the amount of procured reserves from PrM2 model is larger than in the stochastic model despite that a part of those are not fully activated in real-time. However, this procurement schedule allows to modify the day-ahead merit order and eventually reduce expected system cost. This alternative reserve provision schedule shows the ability of model PrM2 to consider both the relationship between energy and reserves as well as the structure of the forecast uncertainty in order to change the geographical distribution and the magnitude of reserve requirements.

Table 8: Area reserve requirements reported from StochM and PrM2 models (values in $\mathrm{MW}$ ).

\begin{tabular}{cccccc}
\hline Model & \multicolumn{2}{c}{ StochM } & & \multicolumn{2}{c}{ PrM2 } \\
\hline & $R R^{+}$ & $R R^{-}$ & & $R R^{+}$ & $R R^{-}$ \\
\cline { 2 - 3 } \cline { 5 - 6 } Area 1 & 0 & 10 & & 0 & 32.5 \\
Area 2 & 4.5 & 25 & & 15.8 & 13.7 \\
\hline
\end{tabular}

In order to emphasize the contribution of decision-support tools to the efficient operation of flexible assets, we compare the market outcome of two setups with interconnections $\left(n_{2}, n_{4}\right)$ and $\left(n_{3}, n_{6}\right)$ being either HVDC or AC lines with capacity of $15 \mathrm{MW}$. The rest of system data remain unchanged. Table 9 presents the expected cost for the two system configurations according to $S e q M$ and PrM1 transmission allocation models. The myopic perception of SeqM model regarding wind power uncertainty and market dynamics, fails to exploit the flexibility of HVDC transmission resulting in higher expected cost 
than the AC setup. In this case, releasing the whole available HVDC capacity at the day-ahead market reduces scheduling cost but increases significantly the balancing cost compared to the more restricted AC setup. On the contrary, the anticipative features of PrM1 allow to exploit better the flexibility of HVDC tie-lines and reduce expected system cost, since the properties and the topology of the network are inherently considered in the transmission allocation process. Note that both models use the same area reserve requirements that enables their fair comparison.

Table 9: Comparison of expected system cost for HVDC vs. AC tie-lines with transmission capacity of $15 \mathrm{MW}(\$)$.

\begin{tabular}{|c|c|c|c|c|c|c|c|c|}
\hline \multirow[t]{2}{*}{ Model } & \multicolumn{4}{|c|}{ HVDC tie-lines } & \multicolumn{4}{|c|}{ AC tie-lines } \\
\hline & $\mathrm{RC}$ & DA & BA & Total & $\mathrm{RC}$ & DA & BA & Total \\
\hline SeqM & 409.0 & 8104.0 & 1615.5 & 10128.5 & 409.0 & 8134.0 & 1281.5 & 9824.5 \\
\hline $\operatorname{PrM1}$ & 401.5 & 8116.5 & -302.0 & 8216.0 & 400.9 & 8176.8 & -229.5 & 8348.2 \\
\hline
\end{tabular}

\subsection{Case study}

We now consider a two-area power system that comprises areas 1 and 2 based on the modernized version of the IEEE Reliability Test System presented in [46]. Each area includes 24 nodes, 32 generators and 35 transmission lines. In addition, area 1 and 2 include nine and six wind farms, respectively. The nodal position, type, generation capacity and energy offers submitted in the dayahead market from conventional and wind power generators are provided in [12]. The two areas are interconnected with three HVDC tie-lines. The network topology and the transmission line parameters are also provided in [12]. Due to their limited flexibility, nuclear, coal and integrated gasification combined cycle (IGCC) units do not provide reserves, whereas open and combined cycle gas turbines (OCGT and CCGT) offer $50 \%$ and 30\% of their capacity for reserve provision at a cost equal to $20 \%$ of their day-ahead energy offer. Wind power production is modeled using a set of 30 equiprobable scenarios that respect the spatial correlation of forecast errors over the different wind farm locations (online available at [9]). The demand at each node is considered inelastic and can be curtailed at a cost of $\$ 1000 / \mathrm{MWh}$ (value of lost load).

All optimization problems were implemented in Python 2.7 using the Gurobi solver package on a Windows-based personal computer Intel(R) Core(TM) i5 with four processors clocking at $2.3 \mathrm{GHz}$ and $8 \mathrm{~GB}$ of RAM. The computational time for $S e q M$ and StochM models was below ten seconds. Models PrM1 and PrM2 were solved to a $0.1 \%$ Benders gap and solution time was kept below four minutes for all instances.

Table 10 presents the total expected cost of the $S e q M$ transmission allocation model for increasing wind power penetration levels, defined as the ratio 
between the total expected wind power production and the system demand. The inter-area HVDC transmission capacity is fully allocated to energy exchange, i.e., $\chi=0$. Higher shares of cost-free wind power production reduce the total operating cost of the system. Reserve requirements are calculated assuming availability of probabilistic forecasts according to the methodology described in Section 4.1. This approach ensures high reliability level in the expense of potential over-procurement of reserves. Hence, the probability that the system has to resort to very expensive load shedding is very low, i.e., only in case where operating reserves cannot be deployed due to network limitations. The approach can be easily adjusted to different reliability levels, e.g., the $95 \%$ interval around the median of wind power predictive distribution, accounting for the trade-off between reserve procurement cost and system reliability. In any case, every deterministic reserve rule remains a heuristic and thus it should not affect the general ranking of the different transmission allocation models presented in this work.

Table 10: Total expected cost (values in $\$$ ) of the $S e q M$ transmission allocation model for different wind power penetration levels.

\begin{tabular}{lcccccccc}
\hline $\begin{array}{l}\text { Wind power } \\
\text { penetration [\%] }\end{array}$ & 15 & 20 & 25 & 30 & 35 & 40 & 45 & 50 \\
\hline Reserve capacity & 1185 & 1583 & 1984 & 2393 & 2807 & 3265 & 3782 & 4339 \\
Day-ahead & 84384 & 77218 & 70305 & 63425 & 56997 & 51581 & 46683 & 42093 \\
Exp. Balancing & -334 & -7 & -30 & 34 & 182 & -10 & -577 & -1179 \\
Total cost & 85235 & 78794 & 72259 & 65852 & 59988 & 54836 & 49888 & 45253 \\
\hline
\end{tabular}

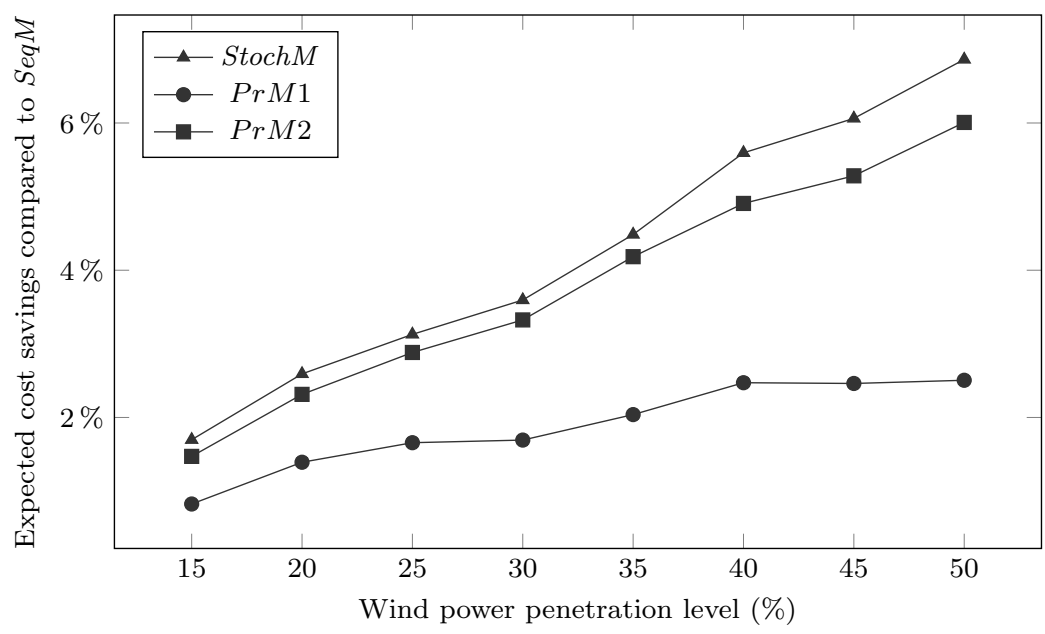

Fig. 5: Expected cost savings of the StochM, PrM1 and PrM2 transmission allocation models compared to SeqM model. 


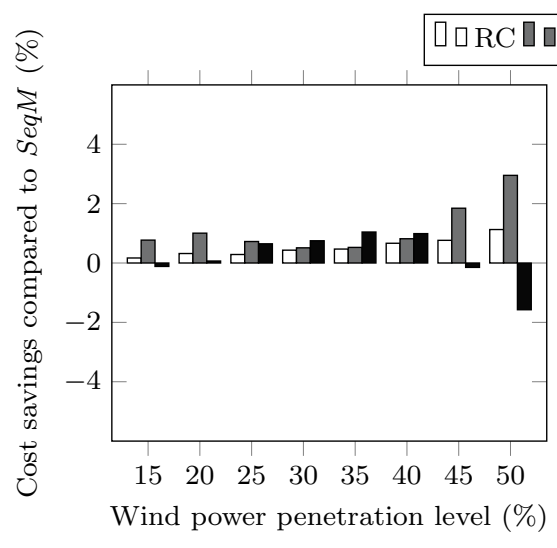

(a) Model $\operatorname{PrM1}$

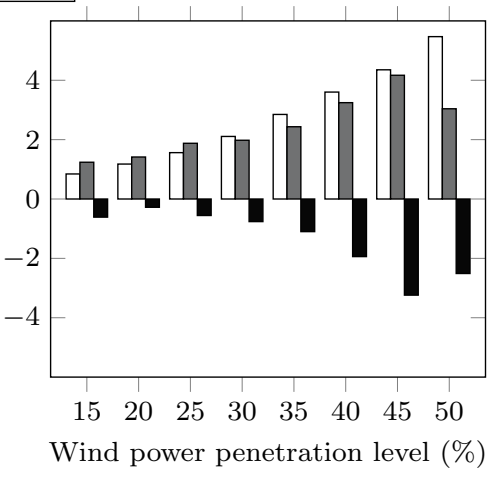

(b) Model PrM2

Fig. 6: Breakdown of total expected cost savings compared to $S e q M$ transmission allocation model among the reserve capacity (RC), day-ahead (DA) and balancing (BA) markets.

The expected savings in total system cost resulting from the more advanced transmission allocation models are presented in Figure 5 as a function of wind power penetration. It can be noticed that all models can reduce expected system cost compared to the status quo $\operatorname{Seq} M$ approach, especially for higher shares of wind power production. Considering as the only degree of freedom the inter-area transmission allocation $\chi$, model PrM1 reduces system cost by about $2 \%$ for wind power shares of $40 \%$, while beyond this level there is a saturation of expected savings. Adding as extra control parameter the area reserve requirements, model PrM2 is able to improve expected cost savings and actually capture most of the benefits of the ideal StochM model.

Aiming to gain a better insight on the distribution of cost savings among the different trading floors, Figures $6 \mathrm{a}$ and $6 \mathrm{~b}$ provide a breakdown of the total expected savings to the reserve capacity, day-ahead and balancing markets for models PrM1 and PrM2, respectively. Here, cost savings for each trading floor are calculated relative to the cost of the $S e q M$ model for the corresponding trading floor. Having as fixed parameter the upward and downward area reserve requirements, model PrM1 (see Figure 6a) still manages to reduce the reserve provision cost by enabling access to inter-area reserve capacity through proper allocation of tie-line capacities. Figure 7 shows the optimal allocation of interconnection capacity for the exchange of reserves $(\chi \%)$ and energy $(1-\chi \%)$ for model PrM1 (left bars). It is important to note that the share of transmission capacity allocated to each service changes significantly with respect to the level of wind power penetration. This effect underlines the importance of dynamic allocation based on a preemptive methodology that accounts for the system parameters, i.e., wind power uncertainty, and the market dynamics. 


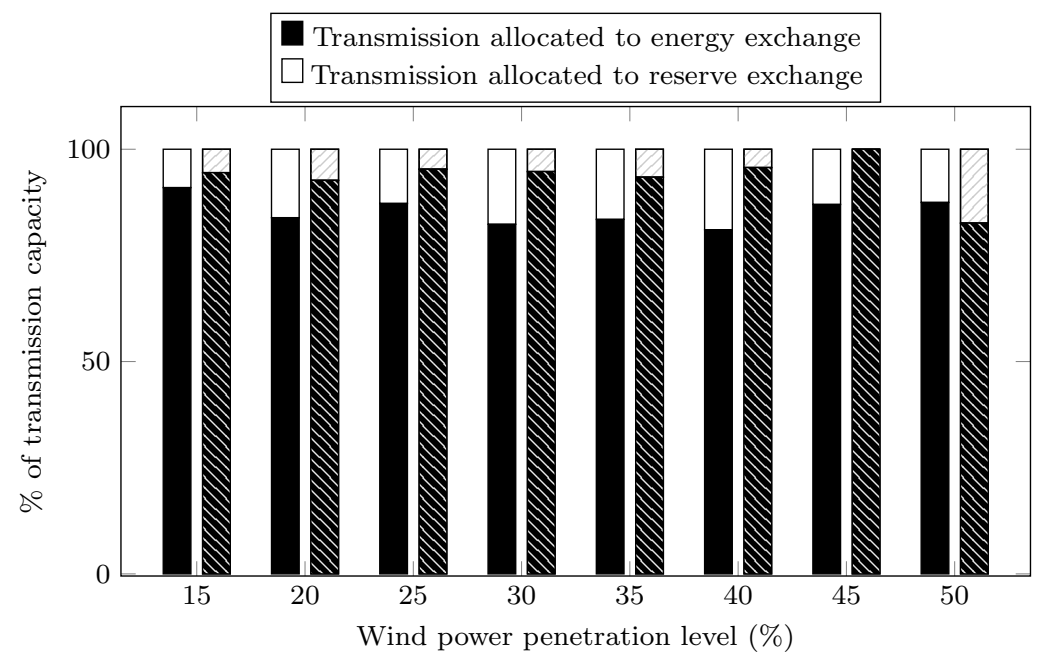

Fig. 7: Transmission capacity allocation between energy and reserves for different wind power penetration levels according to PrM1 (left bars) and PrM2 (right bars) models.

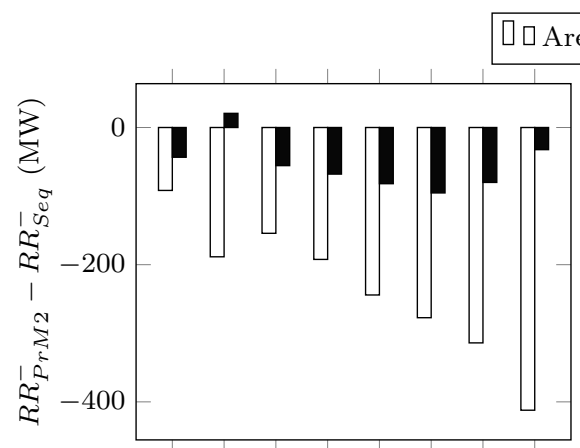

$\begin{array}{llllllll}15 & 20 & 25 & 30 & 35 & 40 & 45 & 50\end{array}$

Wind power penetration level (\%)

(a) Downward reserves $\left(R R^{-}\right)$

\section{Area I】 Area II}

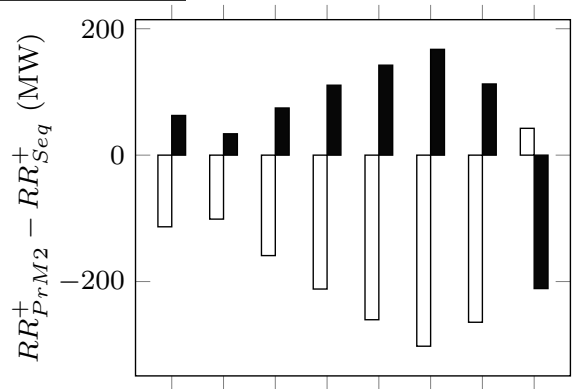

$\begin{array}{llllllll}15 & 20 & 25 & 30 & 35 & 40 & 45 & 50\end{array}$

Wind power penetration level $(\%)$

(b) Upward reserves $\left(R R^{+}\right)$

Fig. 8: Difference in reserve requirements and their geographical distribution between $S e q M$ and PrM2 transmission allocation models.

Enabling also the optimization of area reserve requirements, model PrM2 results in higher cost savings for the reserve capacity and day-ahead markets for the whole range of wind power penetration, as shown in Figure 6b. These savings are partially offset by increased expected cost in real-time operation. Nonetheless, expected balancing cost is only a small fraction of the total cost (see also Table 10) and thus the net benefit for the system is still positive. This extra degree of freedom leads to different allocation of inter-area transmission 
capacity, compared to PrM1 model as shown in Figure 7 (right bars), which also depends dynamically upon wind power penetration. It can be noticed that for most of the cases, PrM2 model makes available higher percentage of transmission for energy trade by reserving lower $\chi \%$ for reserve exchange. This is an effect of the reserve sharing policy that PrM2 model actually implements through the coordinated dimensioning of reserves. As presented in Figures $8 \mathrm{a}$ and $8 \mathrm{~b}$, this policy results in lower upward and downward reserve requirements compared to the SeqM model as well as in their significantly different geographical distribution among the two areas of the system.

\section{Conclusions}

This paper considered the optimal allocation of inter-area HVDC tie-lines for the exchange of energy and reserves in the presence of uncertain power supply. We used as reference the current market design, where reserve capacity, day-ahead and balancing markets are cleared sequentially based on the explicit definition of area reserve requirements and interconnections' allocation between the two services. Against this paradigm, we set up a stochastic energy and reserves co-optimization model that constitutes the ideal benchmark for our study. To bridge the efficiency gap between these two setups, we proposed a preemptive transmission allocation model that bears the anticipativity features of the stochastic approach, while being compatible with the current market structure. The proposed model was formulated as a stochastic bilevel program and recasted as a MILP. Exploiting the structure of the problem at hand, we applied a Benders decomposition strategy to reduce computational burden, ensuring also convergence to the global optimal solution.

Our analysis showed that the current market design can approach the stochastic ideal solution, if certain market parameters are set to appropriate values. For moderate penetration levels of stochastic power generation, the 'smart' tuning of the inter-area capacity allocation between energy and reserves can promptly reduce expected operational cost. The optimal setting of this parameter requires a minimum degree of coordination among operators at adjacent regions akin to the reserve exchange setup. For increasing shares of stochastic production, though, operators must exploit as additional degree of coordination the joint dimensioning and procurement of reserves to enhance market efficiency. Our model can readily incorporate such a reserve sharing policy, optimizing area reserve requirements at both ends of the interconnections. Adopting this type of decision-support tools can capture to a great extent the benefits of stochastic dispatch models, sidestepping some of its practical drawbacks, i.e., pricing issues and consensus on probabilistic information sharing, albeit without radical changes of the existing market setup.

Future work may focus on the development of decentralized optimization schemes that enable the coordination of neighboring areas in terms of reserves sharing and transmission allocation with minimal exchange of intra-area information. This extension will facilitate the cooperation of power system opera- 
tors in adjacent regions while preserving their autonomy and privacy on their domestic network. Another potential future research direction is to consider a multi-period network-constrained electricity pool to include ramping limits or other relevant time-coupling constraints. Furthermore, this model can be extended to consider different control parameters, such as the day-ahead transmission capacity setting of AC lines equipped with 'dynamic line rating' technology. Finally, modeling the impact of strategic behavior of market participants on optimal transmission allocation would be particularly interesting.

\section{Appendix A}

This Appendix provides the complete set of KKT conditions for the reserve capacity and day-ahead market clearing problems (9d) and (9e), respectively, that appear in the lower level of the preemptive allocation model (9). The dual multipliers of inequality constraints are listed to their right with complementarity relationships indicated by the $\perp$ symbol. For equality constraints, the corresponding dual multipliers are indicated after a colon.

The KKT conditions of reserve capacity auction (9d) are:

$$
\begin{aligned}
& 0 \leq R_{i}^{+}-\sum_{a} r_{i a}^{+} \perp \nu_{i}^{\mathrm{R}^{+}} \geq 0, \quad \forall i, \\
& 0 \leq R_{i}^{-}-\sum_{a} r_{i a}^{-} \perp \nu_{i}^{\mathrm{R}^{-}} \geq 0, \quad \forall i, \\
& 0 \leq \sum_{i} r_{i a}^{+}-R R_{a}^{+} \perp \nu_{a}^{\mathrm{RR}} \geq 0, \quad \forall a, \\
& 0 \leq \sum_{i} r_{i a}^{-}-R R_{a}^{-} \perp \nu_{a}^{\mathrm{RR}} \geq 0, \quad \forall a, \\
& 0 \leq \chi_{e} T_{e}-\sum_{i \in \mathcal{M}_{a_{s}(e)}^{I}} r_{i a_{r}(e)}^{+} \perp \xi_{e}^{\mathrm{r}+} \geq 0, \quad \forall e, \\
& 0 \leq \chi_{e} T_{e}-\sum_{i \in \mathcal{M}_{a_{r}(e)}^{I}} r_{i a_{s}(e)}^{+} \perp \xi_{e}^{\mathrm{s}+} \geq 0, \quad \forall e, \\
& 0 \leq \chi_{e} T_{e}-\sum_{i \in \mathcal{M}_{a_{s}(e)}^{I}} r_{i a_{r}(e)}^{-} \perp \xi_{e}^{\mathrm{r}-} \geq 0, \quad \forall e, \\
& 0 \leq \chi_{e} T_{e}-\sum_{i \in \mathcal{M}_{a_{r}(e)}^{I}} r_{i a_{s}(e)}^{-} \perp \xi_{e}^{\mathrm{s}-} \geq 0, \quad \forall e, \\
& 0 \leq C_{i}^{+}+\nu_{i}^{\mathrm{R}^{+}}-\nu_{a}^{\mathrm{RR}}+\xi_{e}^{\mathrm{r}+} \Psi_{a_{s}(e)}^{I}+\xi_{e}^{\mathrm{s}+} \Psi_{a_{r}(e)}^{I} \perp r_{i a}^{+} \geq 0, \forall i, \forall a, \\
& 0 \leq C_{i}^{-}+\nu_{i}^{\mathrm{R}^{-}}-\nu_{a}^{\mathrm{RR}}+\xi_{e}^{\mathrm{r}-} \Psi_{a_{s}(e)}^{I}+\xi_{e}^{\mathrm{s}-} \Psi_{a_{r}(e)}^{I} \perp r_{i a}^{-} \geq 0, \forall i, \forall a,
\end{aligned}
$$

where mapping $\Psi_{a_{s}(e)}^{I}\left(\Psi_{a_{r}(e)}^{I}\right)$ is equal to 1 if unit $i$ is located in the sending (receiving) end of link $e$ and 0 otherwise. 
The KKT conditions of day-ahead market auction (9e) are:

$$
\begin{aligned}
& \sum_{j \in \mathcal{M}_{n}^{J}} w_{j}+\sum_{i \in \mathcal{M}_{n}^{I}} p_{i}-d_{n}-\sum_{\ell \in L^{\mathrm{AC}}} f_{\ell}+\sum_{\ell \in L^{\mathrm{DC}}} A_{\ell n} z_{\ell}=0: \lambda_{n} \text { free, } \forall n \\
& 0 \leq p_{i}-\sum_{a} \hat{r}_{i a}^{-} \perp \nu_{i}^{\mathrm{PR}-} \geq 0, \quad \forall i, \\
& 0 \leq \sum_{a} \hat{r}_{i a}^{+}-p_{i} \perp \nu_{i}^{\mathrm{PR}+} \geq 0, \quad \forall i, \\
& 0 \leq w_{j}-\bar{W}_{j} \perp \nu_{j}^{\mathrm{W}} \geq 0, \quad \forall j, \\
& f_{\ell}=B_{\ell} \sum_{n} A_{\ell n} \delta_{n}: \lambda_{n}^{\mathrm{F}} \text { free, } \quad \forall l \in L^{\mathrm{AC}}, \\
& 0 \leq\left(1-\chi_{\ell}\right) T_{\ell}+f_{\ell} \perp \underline{\xi}_{\ell}^{\mathrm{AC}} \geq 0, \quad \forall \ell \in L^{\mathrm{AC}}, \\
& 0 \leq f_{\ell}-\left(1-\chi_{\ell}\right) T_{\ell} \perp \bar{\xi}_{\ell}^{\mathrm{AC}} \geq 0, \quad \forall \ell \in L^{\mathrm{AC}}, \\
& 0 \leq\left(1-\chi_{\ell}\right) T_{\ell}+z_{\ell} \perp \underline{\xi}_{\ell}^{\mathrm{DC}} \geq 0, \quad \forall \ell \in L^{\mathrm{DC}}, \\
& 0 \leq z_{\ell}-\left(1-\chi_{\ell}\right) T_{\ell} \perp \bar{\xi}_{\ell}^{\mathrm{DC}} \geq 0, \quad \forall \ell \in L^{\mathrm{DC}} \text {, } \\
& \delta_{1}=0: \lambda^{\text {ref }} \text { free, } \\
& 0 \leq C_{i}+\sum_{i \in \mathcal{M}_{n}^{I}} \lambda_{n}+\nu_{i}^{\mathrm{PR}+}-\nu_{i}^{\mathrm{PR}-} \perp p_{i} \geq 0, \quad \forall i, \\
& 0 \leq \sum_{j \in \mathcal{M}_{n}^{J}} \lambda_{n}+\nu_{j}^{\mathrm{W}} \perp w_{j} \geq 0, \quad \forall j \\
& 0 \leq \sum_{n} A_{\ell n} \lambda_{n}+\lambda_{n}^{\mathrm{F}}-\underline{\xi}_{\ell}^{\mathrm{AC}}+\bar{\xi}_{\ell}^{\mathrm{AC}} \perp f_{\ell} \geq 0, \quad \forall \ell \in L^{\mathrm{AC}}, \\
& 0 \leq \sum_{n} A_{\ell n} \lambda_{n}-\underline{\xi}_{\ell}^{\mathrm{DC}}+\bar{\xi}_{\ell}^{\mathrm{DC}} \perp z_{\ell} \geq 0, \quad \forall \ell \in L^{\mathrm{DC}}, \\
& 0 \leq-\sum_{\ell i n L^{\mathrm{AC}}} B_{\ell} A_{\ell n} \lambda_{n}^{\mathrm{F}} \perp \delta_{n} \geq 0, \forall n \backslash n=1, \\
& 0 \leq-\sum_{\ell i n L^{\mathrm{AC}}} B_{\ell} A_{\ell n} \lambda_{n}^{\mathrm{F}}+\lambda^{\mathrm{ref}} \perp \delta_{1} \geq 0, n=1 .
\end{aligned}
$$


Appendix B

Table 11: Power flows for the SeqM and StochM transmission allocation models (values in MW).

\begin{tabular}{lcccccccc}
\hline Model & \multicolumn{3}{c}{$S e q M$} & & \multicolumn{3}{c}{ StochM } \\
\hline \multirow{2}{*}{ Line } & DA & \multicolumn{2}{c}{ BA } & & DA & \multicolumn{2}{c}{ BA } \\
\cline { 2 - 4 } \cline { 7 - 8 }$\left(n_{1}, n_{2}\right)$ & 25 & 20 & 20 & & 20 & 28.4 & 20 \\
\cline { 3 - 4 }$\left(n_{1}, n_{3}\right)$ & 95 & 100 & 100 & & 100 & 91.6 & 100 \\
$\left(n_{2}, n_{3}\right)$ & 70 & 80 & 80 & & 80 & 63.2 & 80 \\
$\left(n_{4}, n_{5}\right)$ & 25 & 22.7 & 33.3 & & 31.8 & 35.3 & 33.3 \\
$\left(n_{4}, n_{6}\right)$ & 75 & 84.8 & 66.7 & & 68.2 & 100 & 66.7 \\
$\left(n_{5}, n_{6}\right)$ & 50 & 62.2 & 33.3 & & 36.3 & 64.8 & 33.3 \\
$\left(n_{2}, n_{4}\right)$ & -20 & -12.5 & -20 & & -20 & 15.2 & -20 \\
$\left(n_{3}, n_{5}\right)$ & -20 & 10 & -20 & & 17.5 & -7.8 & -20 \\
\hline
\end{tabular}

Table 12: Power flows for the PrM1 and PrM2 transmission allocation models (values in MW).

\begin{tabular}{|c|c|c|c|c|c|c|}
\hline \multirow{3}{*}{$\begin{array}{l}\text { Model } \\
\text { Line }\end{array}$} & \multicolumn{3}{|c|}{ PrM1 } & \multicolumn{3}{|c|}{ PrM2 } \\
\hline & \multirow[t]{2}{*}{$\mathrm{DA}$} & \multicolumn{2}{|c|}{$\mathrm{BA}$} & \multirow[t]{2}{*}{$\mathrm{DA}$} & \multicolumn{2}{|c|}{$\mathrm{BA}$} \\
\hline & & $s_{1}$ & $s_{2}$ & & $s_{1}$ & $s_{2}$ \\
\hline$\left(n_{1}, n_{2}\right)$ & 25.8 & 28 & 20 & 20 & 28.4 & 20 \\
\hline$\left(n_{1}, n_{3}\right)$ & 94.2 & 92 & 100 & 100 & 91.6 & 100 \\
\hline$\left(n_{2}, n_{3}\right)$ & 68.3 & 64 & 80 & 80 & 63.2 & 80 \\
\hline$\left(n_{4}, n_{5}\right)$ & 25.8 & 34 & 33.3 & 32.1 & 35.3 & 33.3 \\
\hline$\left(n_{4}, n_{6}\right)$ & 76.7 & 100 & 66.7 & 77.9 & 100 & 66.7 \\
\hline$\left(n_{5}, n_{6}\right)$ & 50.8 & 66 & 33.3 & 45.8 & 64.8 & 33.3 \\
\hline$\left(n_{2}, n_{4}\right)$ & -17.5 & 14 & -20 & -10 & 15.3 & -20 \\
\hline$\left(n_{3}, n_{5}\right)$ & -17.5 & -9 & -20 & 2.5 & -7.8 & -20 \\
\hline
\end{tabular}




\section{Nomenclature}

The main notation used in this paper is stated below. Additional symbols are defined in the paper when needed.

\section{Sets}

A Set of areas.

$a_{r}(e) \quad$ Receiving-end area of link $e$.

$a_{s}(e) \quad$ Sending-end area of link $e$.

$E \quad$ Set of inter-area links $e$.

I Set of dispatchable power plants.

$J \quad$ Set of stochastic power plants.

$L^{\mathrm{AC}} \quad$ Set of AC transmission lines.

$L^{\mathrm{DC}} \quad$ Set of HVDC transmission lines.

$N \quad$ Set of network nodes.

$\mathcal{M}_{n}^{I} \quad$ Set of dispatchable power plants $i$ located at node $n$.

$\mathcal{M}_{n}^{J} \quad$ Set of stochastic power plants $j$ located at node $n$.

$\mathcal{M}_{a}^{I} \quad$ Set of dispatchable power plants $i$ located in area $a$.

$\Lambda_{a_{r}(e)}^{a_{s}(e)} \quad$ Set of AC and HVDC lines connecting areas $a_{s}(e)$ and $a_{r}(e)$ across link $e$.

$\mathcal{S} \quad$ Set of stochastic power production scenarios.

\section{Parameters}

$\bar{W}_{j} \quad$ Expected power production of stochastic power plant $j[\mathrm{MW}$.

$\pi_{s} \quad$ Probability of occurrence of scenario $s$.

$A_{\ell n} \quad$ Line-to-bus incidence matrix.

$B_{\ell} \quad$ Absolute value of the susceptance of AC line $\ell$ [per unit].

$D_{n} \quad$ Demand at node $n[\mathrm{MW}]$.

$C_{i} \quad$ Energy offer price of power plant $i[\$ / \mathrm{MWh}]$.

$C_{i}^{+/-} \quad \mathrm{Up} /$ down reserve capacity offer price of power plant $i$ [\$/MW].

$C^{\text {sh }} \quad$ Value of involuntarily shed load [\$/MWh].

$P_{i} \quad$ Capacity of dispatchable power plant $i[\mathrm{MW}]$.

$R_{i}^{+/-} \quad \mathrm{Up} /$ down reserve capacity offer quantity of power plant $i[\$ / \mathrm{MW}$.

$R R_{a}^{+/-} \mathrm{Up} /$ down reserve capacity requirements of area $a[\mathrm{MW}]$.

$T_{e / \ell} \quad$ Transmission capacity of link $e /$ line $\ell[\mathrm{MW}]$.

$W_{j s} \quad$ Power production by stochastic power plant $j$ in scenario $s[\mathrm{MW}$.

\section{Variables}

$\delta_{n} \quad$ Voltage angle at node $n$ at day-ahead stage [rad].

$\tilde{\delta}_{n} \quad$ Voltage angle at node $n$ in scenario $s$ [rad].

$\chi_{e / \ell} \quad$ Percentage of inter-area interconnection capacity of link $e /$ line $\ell$ allocated to reserves exchange.

$f_{\ell} \quad$ Power flow in AC line $\ell$ at day-ahead stage [MW].

$\tilde{f}_{\ell s} \quad$ Power flow in AC line $\ell$ in scenario $s$ [MW].

$l_{n s}^{\text {sh }} \quad$ Load shedding at node $n$ in scenario $s[\mathrm{MW}]$. 
$p_{i} \quad$ Day-ahead schedule of dispatchable power plant $i[\mathrm{MW}$.

$p_{i s}^{+/-} \quad \mathrm{Up} /$ down regulation provided by dispatchable power plant $i$ in scenario $s[\mathrm{MW}]$.

$r_{i a}^{+/-} \quad \mathrm{Up} /$ down reserve capacity scheduled for dispatchable power plant $i$, procured by area $a[\mathrm{MW}]$.

$w_{j} \quad$ Day-ahead schedule of stochastic power plant $j[\mathrm{MW}]$.

$w_{j s}^{\text {spill }} \quad$ Power spilled by stochastic power plant $j$ in scenario $s[\mathrm{MW}]$.

$z_{\ell} \quad$ Power flow in HVDC line $\ell$ at day-ahead stage [MW].

$\tilde{z}_{\ell s} \quad$ Power flow in HVDC line $\ell$ in scenario $s[\mathrm{MW}]$.

\section{References}

1. Agency for the Cooperation of Energy Regulators (ACER): Framework guidelines on electricity balancing (2012). URL http://www.acer.europa.eu/official_documents/ acts_of_the_agency/framework_guidelines/framework\%20guidelines/framework\% 20 guidelines $\% 20$ on $\% 20$ electricity $\% 20$ balancing.pdf

2. Ahlstrom, M., Ela, E., Riesz, J., O’Sullivan, J., Hobbs, B.F., O’Malley, M., Milligan, M., Sotkiewicz, P., Caldwell, J.: The evolution of the market: Designing a market for high levels of variable generation. IEEE Power and Energy Magazine 13(6), 60-66 (2015)

3. Bahrman, M., Johnson, B.: The ABCs of HVDC transmission technologies. Power and Energy Magazine, IEEE 5(2), 32-44 (2007). DOI 10.1109/MPAE.2007.329194

4. Baldick, R., Helman, U., Hobbs, B.F., O'Neill, R.P.: Design of efficient generation markets. Proceedings of the IEEE 93(11), 1998-2012 (2005)

5. Van den Bergh, K., Broder, R., Delarue, E., D'haeseleer, W., Hobbs, B.: The benefits of coordinating the sizing, allocation and activation of flexibility between market zones. TME working paper 2015-14 (2015)

6. Birge, J.R., Louveaux, F.: Introduction to stochastic programming. Springer Science \& Business Media (2011)

7. Biskas, P.N., Chatzigiannis, D.I., Bakirtzis, A.G.: European electricity market integration with mixed market designs-Part I: Formulation. IEEE Transactions on Power Systems 29(1), 458-465 (2014)

8. Bondy, D.E.M., Tarnowski, G., Heussen, K., Hansen, L.H.: Operational scenario: Manual regulating power. Tech. rep., iPower Consortium (2014)

9. Bukhsh, W.: Data for stochastic multiperiod optimal power flow problem. URL https: //sites.google.com/site/datasmopf/

10. Conejo, A.J., Castillo, E., Minguez, R., Garcia-Bertrand, R.: Decomposition techniques in mathematical programming: engineering and science applications. Springer Science \& Business Media (2006)

11. De Vos, K., Morbee, J., Driesen, J., Belmans, R.: Impact of wind power on sizing and allocation of reserve requirements. IET Renewable Power Generation 7(1), 1-9 (2013)

12. Delikaraoglou, S., Pinson, P.: Electronic companion: System data - Optimal allocation of HVDC interconnections for exchange of energy and reserve capacity services. URL http://doi.org/10.5281/zenodo.885250

13. Delikaraoglou, S., Pinson, P., Eriksson, R., Weckesser, J.T.G.: Optimal dynamic capacity allocation of HVDC interconnections for cross-border exchange of balancing services in presence of uncertainty. In: CIGRE International Symposium: Across borders - HVDC systems and market integration, pp. 1-7. CIGRE (2015)

14. Doherty, R., O'Malley, M.: A new approach to quantify reserve demand in systems with significant installed wind capacity. IEEE Transactions on Power Systems 20(2), 587-595 (2005)

15. Ela, E., Milligan, M., Kirby, B.: Operating reserves and variable generation. NREL (2011) 
16. Energinet.dk: Reservation of capacity for ancillary services - general principles and the SK4 case (2010). URL http://www.elforsk.se/Documents/Market\%20Design/seminars/ Interconnectors/4_Energinetdk.pdf

17. ENTSO-E: Network code on load-frequency control and reserves (2013). URL https://www.entsoe.eu/fileadmin/user_upload/_library/resources/LCFR/130628NC_LFCR-Issue1.pdf

18. ENTSO-E: Network code on load-frequency control and reserves (2013). URL http://www.acer.europa.eu/Official_documents/Acts_of_the_Agency/Annexes/ The $\% 20$ Network $\% 20$ Code $\% 20$ on $\% 20$ Load-Frequency $\% 20$ Control $\% 20$ and $\% 20$ Reserves $\%$ 20submitted $\% 20$ on $\% 2028 \% 20$ June $\% 202013$.pdf

19. ENTSO-E: Supporting document for the network code on electricity balancing (2013). URL https://www.entsoe.eu/Documents/Network\%20codes $\% 20$ documents/NC\% 20EB/131223_NC_EB_Supporting_Document_FINAL.pdf

20. ENTSO-E: Updated draft of the electricity balancing guideline (2015). URL https://ec.europa.eu/energy/sites/ener/files/documents/ informal\_service \_level\_ebgl\_24-01-2017.pdf

21. ERCOT: Methodologies for determining ancillary service requirements (2012). URL www.ercot.com/meetings/wms/keydocs/2004/0819/WMS08192004-3.doc

22. Farahmand, H., Aigner, T., Doorman, G.L., Korpas, M., Huertas-Hernando, D.: Balancing market integration in the northern European continent: A 2030 case study. IEEE Transactions on Sustainable Energy 3(4), 918-930 (2012)

23. Fortuny-Amat, J., McCarl, B.: A representation and economic interpretation of a twolevel programming problem. Journal of the operational Research Society pp. 783-792 (1981)

24. Gabriel, S.A., Conejo, A.J., Fuller, J.D., Hobbs, B.F., Ruiz, C.: Complementarity modeling in energy markets, vol. 180. Springer Science \& Business Media (2012)

25. Galiana, F.D., Bouffard, F., Arroyo, J.M., Restrepo, J.F.: Scheduling and pricing of coupled energy and primary, secondary, and tertiary reserves. Proceedings of the IEEE 93(11), 1970-1983 (2005)

26. Gebrekiros, Y.T.: Analysis of integrated balancing markets in northern Europe under different market design options (2015)

27. Gilbert, R., Neuhoff, K., Newbery, D.: Allocating transmission to mitigate market power in electricity networks. RAND Journal of Economics pp. 691-709 (2004)

28. Hagspiel, S., Papaemannouil, A., Schmid, M., Andersson, G.: Copula-based modeling of stochastic wind power in europe and implications for the swiss power grid. Applied Energy 96, 33-44 (2012)

29. Haubrich, H., Hinüber, G., Mirbach, T., Tersteegen, B.: Technical issues regarding open market coupling-omc. RWTH Aachen University (2006)

30. Hobbs, B.F., Rijkers, F.A., Boots, M.G.: The more cooperation, the more competition? a Cournot analysis of the benefits of electric market coupling. The Energy Journal pp. 69-97 (2005)

31. Holttinen, H., Milligan, M., Ela, E., Menemenlis, N., Dobschinski, J., Rawn, B., Bessa R.J., Flynn, D., Gomez-Lazaro, E., Detlefsen, N.K.: Methodologies to determine operating reserves due to increased wind power. IEEE Transactions on Sustainable Energy 3(4), 713-723 (2012)

32. Jónsson, T., Pinson, P., Madsen, H.: On the market impact of wind energy forecasts. Energy Economics 32(2), 313-320 (2010)

33. Jónsson, T., Pinson, P., Nielsen, H.A., Madsen, H., Nielsen, T.S.: Forecasting electricity spot prices accounting for wind power predictions. IEEE Transactions on Sustainable Energy 4(1), 210-218 (2013)

34. Karangelos, E., Wehenkel, L.: Probabilistic reliability management approach and criteria for power system real-time operation. In: Power Systems Computation Conference (PSCC), 2016, pp. 1-9. IEEE (2016)

35. King, J., Kirby, B., Milligan, M., Beuning, S.: Flexibility reserve reductions from an energy imbalance market with high levels of wind energy in the Western Interconnection. Tech. rep., National Renewable Energy Laboratory (NREL), Golden, CO. (2011)

36. Makarov, Y.V., Loutan, C., Ma, J., De Mello, P.: Operational impacts of wind generation on California power systems. IEEE Transactions on Power Systems 24(2), 1039-1050 (2009) 
37. Matos, M.A., Bessa, R.J.: Setting the operating reserve using probabilistic wind power forecasts. IEEE Transactions on Power Systems 26(2), 594-603 (2011)

38. Min, L., Abur, A.: Total transfer capability computation for multi-area power systems. IEEE Transactions on Power Systems 21(3), 1141-1147 (2006)

39. Mitridati, L., Pinson, P.: A Bayesian inference approach to unveil supply curves in electricity markets. IEEE Transactions on Power Systems (2017)

40. Morales, J.M., Conejo, A.J., Liu, K., Zhong, J.: Pricing electricity in pools with wind producers. IEEE Transactions on Power Systems 27(3), 1366-1376 (2012)

41. Morales, J.M., Zugno, M., Pineda, S., Pinson, P.: Electricity market clearing with improved scheduling of stochastic production. European Journal of Operational Research 235(3), 765-774 (2014)

42. Mott MacDonald: Impact assessment on European electricity balancing market (2013). URL https://ec.europa.eu/energy/sites/ener/files/documents/ 20130610_eu_balancing_master.pdf

43. Neuhoff, K.: Large-scale deployment of renewables for electricity generation. Oxford review of economic policy 21(1), 88-110 (2005)

44. Ordoudis, C., Delikaraoglou, S., Pinson, P., Kazempour, J.: Exploiting flexibility in coupled electricity and natural gas markets: A price-based approach. In: 12th IEEE Power and Energy Society PowerTech Conference. IEEE (2017)

45. Ott, A.L.: Experience with PJM market operation, system design, and implementation. IEEE Transactions on Power Systems 18(2), 528-534 (2003)

46. Pandzic, H., Dvorkin, Y., Qiu, T., Wang, Y., Kirschen, D.: Library of the renewable energy analysis lab (REAL), University of Washington, Seattle, USA. (2014). URL http://www.ee.washington.edu/research/real/library.html

47. Papavasiliou, A., Oren, S.S.: Multiarea stochastic unit commitment for high wind penetration in a transmission constrained network. Operations Research 61(3), 578-592 (2013)

48. Pérez-Arriaga, I.J.: Regulation of the power sector. Springer (2014)

49. Pineda, S., Bylling, H., Morales, J.: Efficiently solving linear bilevel programming problems using off-the-shelf optimization software. Optimization and Engineering 19(1), $187-211(2018)$

50. Pineda, S., Morales, J.M.: Capacity expansion of stochastic power generation under two-stage electricity markets. Computers \& Operations Research 70, 101-114 (2016)

51. Pozo, D., Sauma, E., Contreras, J.: Basic theoretical foundations and insights on bilevel models and their applications to power systems. Annals of Operations Research 254(12), 303-334 (2017)

52. Pritchard, G., Zakeri, G., Philpott, A.: A single-settlement, energy-only electric power market for unpredictable and intermittent participants. Operations research 58(4-part2), 1210-1219 (2010)

53. Ruiz, C., Conejo, A.J., Bertsimas, D.J.: Revealing rival marginal offer prices via inverse optimization. IEEE Transactions on Power Systems 28(3), 3056-3064 (2013)

54. Svenska Kraftnät, Statnett: The hasle-pilot project (2015). URL http:// www.statnett.no/Global/Hasle\%20report\%20StGr_150317\%20(3).pdf

55. Svenska Kraftnät, Statnett: Hasle-pilot project. methodology and parameters for determining capacity reservation. (2015). URL http://www.statnett.no/Global/ Dokumenter/Kraftsystemet/Markedsinformas jon/Frekvensstyrte $\% 20$ og $\% 20$ sekund $\%$ C3\%A6re/MetodeOgParametreTilMarkedet_RN\%20 (3) $\% 20$ (2) $\% 20$ (2) \%20 (3) Ren2.pdf

56. Vicente, L.N., Calamai, P.H.: Bilevel and multilevel programming: A bibliography review. Journal of Global optimization 5(3), 291-306 (1994)

57. Von Stackelberg, H.: Market structure and equilibrium. Springer Science \& Business Media (2010)

58. Zakeri, G., Pritchard, G., Bjorndal, M., Bjorndal, E.: Pricing wind: a revenue adequate, cost recovering uniform auction for electricity markets with intermittent generation. Tech. rep., Working paper, The University of Aukland. Available: www. optimizationonline. org/DB FILE/2016/06/5484. pdf (2016)

59. Zavala, V.M., Kim, K., Anitescu, M., Birge, J.: A stochastic electricity market clearing formulation with consistent pricing properties. Operations Research 65(3), 557-576 (2017)

60. Zugno, M., Pinson, P., Madsen, H.: Impact of wind power generation on European crossborder power flows. IEEE Transactions on Power Systems 28(4), 3566-3575 (2013) 